\title{
基于有机双层的双极性有机场效应晶体管研究进展
}

\author{
李敏吕爱风* \\ (上海工程技术大学化学化工学院 上海 201620)
}

\begin{abstract}
摘要 由双极性有机场效应晶体管(OFETs)制备的有机互补电路具有操作电压低、能耗低和成本低等优点, 在有机互补 电路方面有很大的应用前景, 引起了科学家们极大的研究兴趣. 同时具有高且匹配的空穴迁移率和电子迁移率的双极 性有机半导体分子是制备高性能有机互补电路的必要条件之一, 然而迄今为止该类双极性有机半导体分子的报道比较 少, 大部分双极性有机半导体分子的空穴和电子传输不匹配; 高性能单极性有机半导体分子报道已有成千上万种, 选 择迁移率相匹配的 $\mathrm{n}$ 型有机半导体分子和 $\mathrm{p}$ 型有机半导体分子构筑具有垂直双层导电沟道的有机场效应晶体管是制备 高性能双极性有机场效应晶体管的有效方法. 总结了构筑基于有机双层的高性能双极性有机场效应晶体管的必要条 件, 已报道的有机双层场效应晶体管的电学性能, 以及影响双极性场效应性能的因素.
\end{abstract}

关键词 有机场效应晶体管; 双极性; 有机双层; 匹配; 迁移率

\section{Recent Progress in Ambipolar Organic Field-Effect Transistors Based on Organic Semiconductor Bilayer}

\author{
Li, Min Lv, Aifeng* \\ (School of Chemistry and Chemical Engineering, Shanghai University of Engineering Science, Shanghai 201620)
}

\begin{abstract}
Complementary circuits made of ambipolar organic field-effect transistors (OFETs) have the advantages of low signal to noise ratio, low operation voltage, low power consumption, and so on. Up to now, only a few ambipolar organic semiconductors with balanced hole and electron mobilities have been reported, which is one of the key requirements for high performance complementary circuits. There are thousands of high performance unipolar organic semiconductors. The conducting channels based on both an n-type and a p-type organic semiconductors, which have matched mobilities, will be an effective way to build a high performance ambipolar OFETs. In this review, the key requirements, the electrical properties, and factors correlating with the performance of ambipolar OFETs based on organic bilayer are reported.
\end{abstract}

Keywords organic field-effect transistors (OFETs); ambipolar; organic bilayer; balanced; mobility

与单极性有机场效应晶体管(Organic Field-Effect Transistors, OFETs)相比, 由双极性有机场效应晶体管 制备的有机互补电路操作电压低、能耗低、成本低, 在 有机光电器件的应用前景更大, 引起了科学家们极大的 研究兴趣 ${ }^{[1]}$. 同时具有高的空穴迁移率 $\left(\mu_{\mathrm{h}}\right)$ 和电子迁移 率 $\left(\mu_{\mathrm{e}}\right)$, 且两种迁移率相当的双极性有机半导体材料 (Organic Semiconductors, OSCs) 是获得高性能有机电路 的必要条件. 但是, 双极性有机半导体分子报道比较少, 且迁移率一般比较低 ${ }^{[2]}$, 而高性能单极性有机场效应晶 体管的研究已经取得很大进展, 如空穴迁移率已达到 $20 \mathrm{~cm}^{2} \bullet \mathrm{V}^{-1} \bullet \mathrm{s}^{-1}$ 和电子迁移率已达到 $10 \mathrm{~cm}^{2} \bullet \mathrm{V}^{-1} \bullet \mathrm{s}^{-1[3]}$.
目前为获得高性能的双极性有机场效应晶体管，主要面 临两个挑战：第一，双极性有机场效应晶体管应该具有 匹配的电子迁移率和空穴迁移率; 第二, 电子和空穴从 同一电极的注入效率应该接近 ${ }^{[4]}$.

从已报道的单极性有机半导体中选择合适的 $\mathrm{p}$ 型有 机半导体和 $\mathrm{n}$ 型有机半导体 ${ }^{[5]}$, 然后制备成具有垂直双 层结构的双极性有机场效应晶体管, 能有效克服上述两 大挑战. 选择的两种有机半导体需要具有以下特点: 首 先 $\mathrm{p}$ 型有机半导体的空穴迁移率和 $\mathrm{n}$ 型有机半导体的电 子迁移率要接近, 其次 $\mathrm{p}$ 型有机半导体的最高占据分子 轨道(HOMO)和 $\mathrm{n}$ 型有机半导体的最低未占据分子轨道

* Corresponding author. E-mail: lvaifeng@sues.edu.cn

Received July 6, 2021; revised August 25, 2021; published online September 6, 2021.

Project supported by the National Natural Science Foundation of China (No. 51803118).

国家自然科学基金(No. 51803118)资助项目. 
(LUMO)也要接近, 有利于同时与晶体管金属电极的功 函进行匹配，提高载流子注入效率. Dodabalapur 等 ${ }^{[6]}$ 在 1995 年报道了由 $\mathrm{n}$ 型 $\mathrm{C}_{60}$ 和 $\mathrm{p}$ 型 $\alpha$-六噻吩两种有机半导 体材料制备的双极性有机场效应晶体管，迁移率只有 $10^{-3} \mathrm{~cm}^{2} \cdot \mathrm{V}^{-1} \cdot \mathrm{s}^{-1}$. 随后引起了基于有机双层的双极性 有机场效应晶体管的研究热潮, 目前该类双极性有机场 效应晶体管电子和空穴的迁移率超过 $2.0 \mathrm{~cm}^{2} \bullet \mathrm{V}^{-1} \bullet \mathrm{s}$ ${ }^{-1}[7]$. 此外, 有机半导体层的沉积顺序、有机半导体层的 薄膜厚度和器件结构也会影响有机半导体层和界面中 的陷阱态密度和载流子的注入势垒 ${ }^{[8]}$, 最终影响双极性 有机场效应晶体管的电荷传输性能.

在这篇综述中, 总结了已报道的基于有机双层的双 极性有机场效应晶体管, 并分析总结了 $\mathrm{HOMO} / \mathrm{LUMO}$ 能级、电极材料、有机薄膜厚度和器件结构等因素对场 效应性能的影响. 希望能为致力于研究高性能双极性有 机场效应晶体管的科学家们提供理论参考.

\section{1 基于有机双层的双极性场效应晶体管}

\section{1 基于有机-有机双层的双极性场效应晶体管}

自 Dodabalapur 等 ${ }^{[6,9]}$ 报道以来, 有机双层结构已被 广泛应用于双极性有机场效应晶体管. 双层有机场效应 晶体管的导电沟道通常包含一种 $\mathrm{n}$ 型有机半导体和一种 $\mathrm{p}$ 型有机半导体, 如图 1 所示 ${ }^{[10]}$. 这两种半导体材料一 般是有机小分子, 双层有机层的制备用真空蒸镀方法, 能同时保证有机双层有机膜的有序性 ${ }^{[6,11]}$. 此外, 高性 能有机双层晶体管中有机小分子的共轭结构通常比较 大, 两种有机小分子的迁移率一般都比较高. 有机双层 晶体管中的两种小分子按分子化学结构特点主要分为 共轭骨架相近的和共轭骨架不相近的两大类.

基于共轭骨架相近的两种小分子制备的双极性晶 体管的电学性能一般比较高, 且两种载流子的迁移率一 般在同一个数量级, 比较匹配. 由 $\mathrm{F}_{16} \mathrm{CuPc}(\mathbf{1 D}$, 底
层 $) / \mathrm{CuPc}(1 \mathrm{C}$, 上层)制备的双层有机场效应晶体管, $\mathrm{F}_{16} \mathrm{CuPc}$ 的薄膜厚度为 $2 \mathrm{~nm}$ 时获得了最佳的电学性能, 空穴/电子迁移率相当, 为 $10^{-6} \mathrm{~cm}^{2} \cdot \mathrm{V}^{-1} \cdot \mathrm{s}^{-1}$ (表 1 , 图 $2)^{[9 \mathrm{c}]}$. 由 $\mathrm{F}_{16} \mathrm{CuPc}(\mathbf{1 D}$, 底层 $) / \mathrm{CuPc}(\mathbf{1 C}$, 上层)制成的有 机双层晶体管获得了 $2.96 \times 10^{-3}$ 和 $9.49 \times 10^{-3} \mathrm{~cm}^{2}$ ・ $\mathrm{V}^{-1} \cdot \mathrm{s}^{-1}$ 的空穴迁移率和电子迁移率, 是具有相反沉积 顺序的有机双层晶体管的 10 倍(表 1), 原子力表征显示 $\mathrm{F}_{16} \mathrm{CuPc}$ 薄膜的表面比较平滑, 更有利于上层 $\mathrm{CuPc}$ 薄膜 的有序沉积(图 3) ${ }^{[12]}$. 基于并五苯 $(1 \mathrm{H}$, 底层)/PTCDIC13 (1I, 上层)制备的有机双层场效应晶体管的最佳底 层有机层薄膜厚度是 $12 \mathrm{~nm}$, 获得的空穴迁移率和电子 迁移率相当, 为 0.41 和 $0.40 \mathrm{~cm}^{2} \bullet \mathrm{V}^{-1} \bullet \mathrm{s}^{-1}[13]$. 最近报道 的 TTF-CN (1P, 底层)/C6-DPA (1Q, 上层)双层晶体管 的电子和空穴迁移率也相当, 分别是 0.87 和 $0.82 \mathrm{~cm}^{2}$ $\mathrm{V}^{-1} \cdot \mathrm{s}^{-1}$, 与高性能单极性有机场效应晶体管性能相 当 ${ }^{[14]}$. 已报道的有机双层场效应晶体管如 $\mathrm{CuPc}(1 \mathrm{C}$, 底 层 $) / \mathrm{SnCl}_{2} \mathrm{Pc}(\mathbf{1 J} \text {, 上层 })^{[10]}$, 并五苯 $(\mathbf{1 H}$, 底层 $) / \mathrm{NTFBII}$ $(1 \mathrm{~K} \text {, 上层 })^{[15]}, \mathrm{AC} 5-\mathrm{CF} 3(1 \mathrm{~L}$ ，底层 $) / \mathrm{BP} 3 \mathrm{~T}(\mathbf{1 M}$, 上 层 $)^{[16]}, \mathrm{P} 3 \mathrm{HT}(\mathbf{1} \mathbf{N}$, 底层 $)$ /菜酰亚胺衍生物 NDI-C9 (10, 上层 $)^{[17]}$ 四种双层晶体管的电子和空穴迁移率都匹配, 在同一个数量级(表 1, 图 2).

基于共轭骨架不相近的两种小分子制备的双极性 晶体管, 电学性能一般比较低, 且两种载流子的迁移率 相差较大, 匹配性不好. 如 $\mathrm{C}_{60}(\mathbf{1 B}$, 底层 $) /$ 并五苯 $(\mathbf{1 H}$, 上层)有机双层晶体管是真空蒸镀法制备的(图 2), 获得 的空穴迁移率和电子迁移率分别为 0.2 和 $0.04 \mathrm{~cm}^{2}$ $\mathrm{V}^{-1} \cdot \mathrm{s}^{-1}$, 空穴迁移率是电子迁移率的 5 倍 ${ }^{[18]}$. 在 $\mathrm{C} 10-\mathrm{DNTT}(\mathbf{1 F}$, 底层 $) / \mathrm{F}_{16} \mathrm{CuPc}(\mathbf{1 D}$, 上层)双层有机膜 中(图 2), 先用溶液剪切法沉积结晶度高的 C10-DNTT (1F) 薄膜, 作为上层 $\mathrm{F}_{16} \mathrm{CuPc}(1 \mathrm{D})$ 的生长模板, 获得空 穴迁移率和电子迁移率分别为 23.7 和 $0.06 \mathrm{~cm}^{2} \cdot \mathrm{V}^{-1} \cdot \mathrm{s}^{-1}$, 高结晶 C10-DNTT (1F) 底层薄膜对上层的 $\mathrm{F}_{16} \mathrm{CuPc}$ (1D) (a)

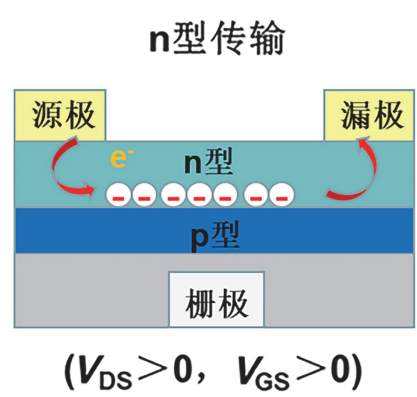

(b)

\section{p 型传输}

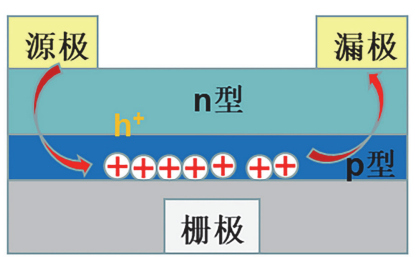

$\left(V_{\mathrm{DS}}<0, \quad V_{\mathrm{GS}}<0\right)$

图 1 基于有机双层的双极性场效应晶体管的两种载流子传输

Figure 1 Charge carrier transport of ambipolar transistors based on organic bilayer

(a) n-Type charge transport and (b) p-type transport. $(-)$ represents the movement of electrons and $(+)$ represents the movement of holes 


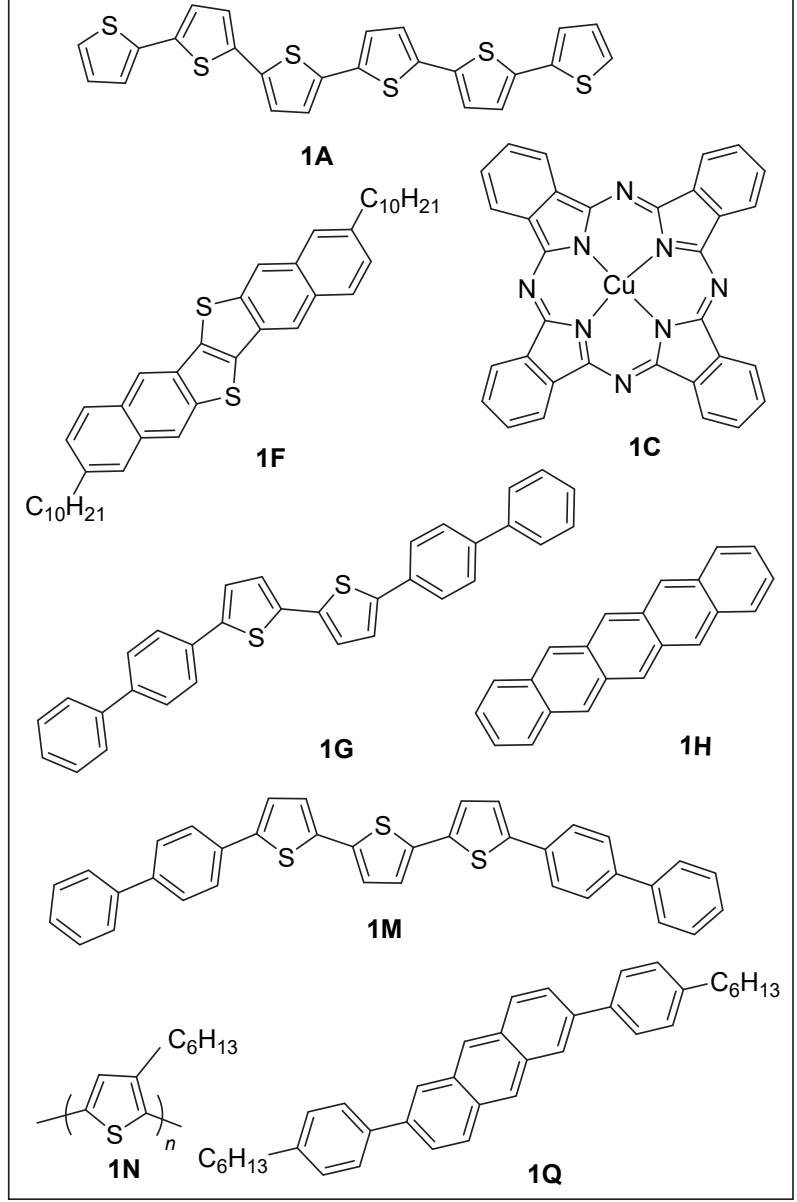

p-type OSCs

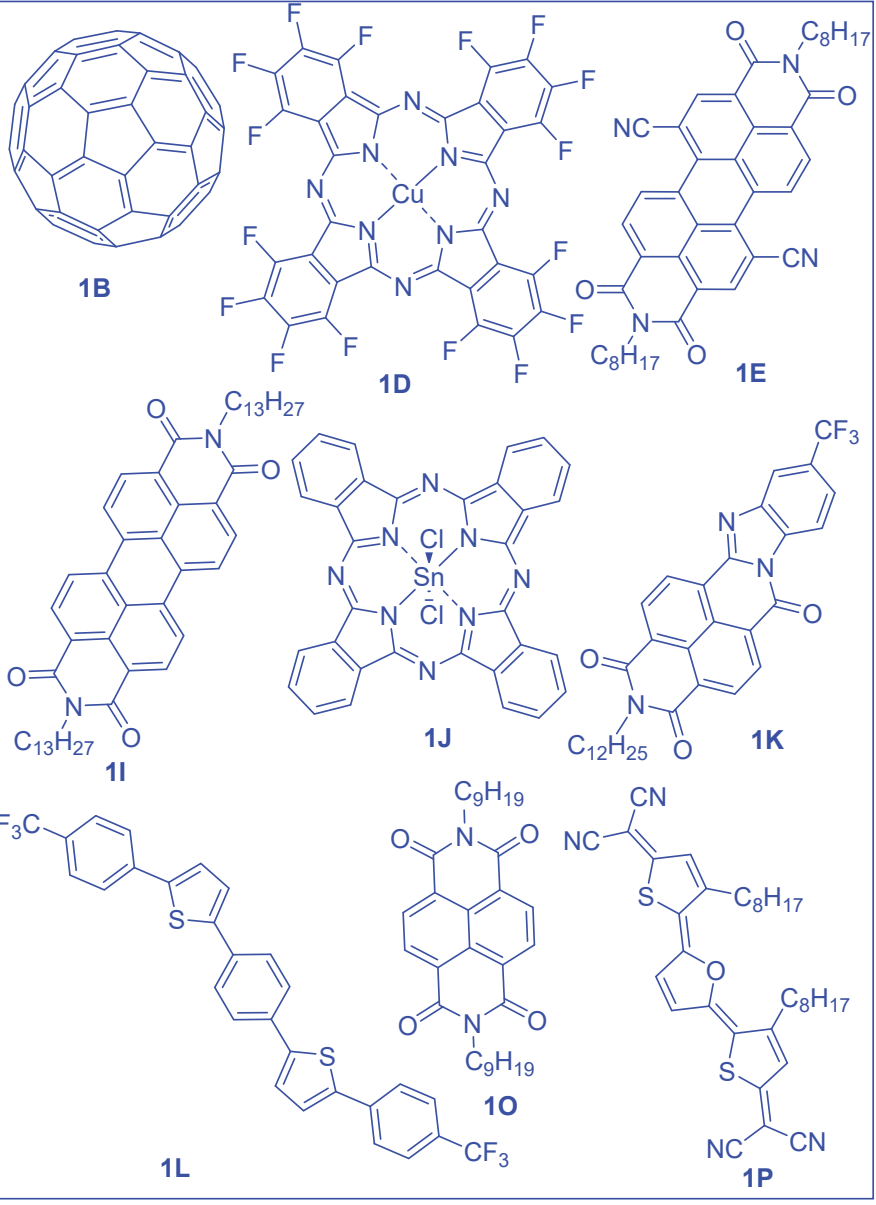

n-type OSCs

图 2 双极性有机场效应晶体管中有机一有机双层的有机半导体分子结构

Figure 2 Molecular structures of OSCs applied in the ambipolar OFETs based on organic-organic bilayer
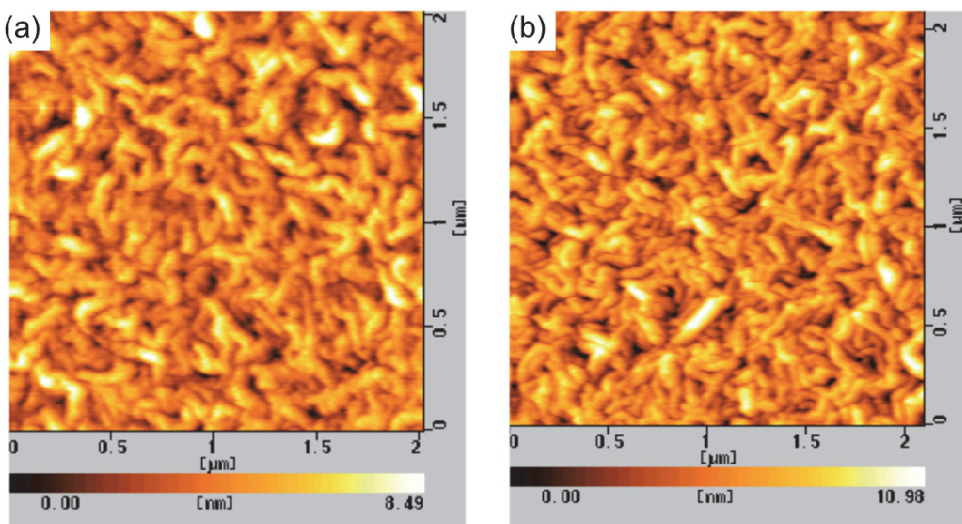

图 $3 \mathrm{~F}_{16} \mathrm{CuPc}(5 \mathrm{~nm}) / \mathrm{CuPc}(10 \mathrm{~nm})$ 有机双层和 $\mathrm{CuPc}(5 \mathrm{~nm}) / \mathrm{F}_{16} \mathrm{CuPc}(15 \mathrm{~nm})$ 有机双层的 $\mathrm{AFM}$ 表征图

Figure 3 AFM morphology of (a) an $\mathrm{F}_{16} \mathrm{CuPc}(5 \mathrm{~nm}) / \mathrm{CuPc}(10 \mathrm{~nm})$ organic bilayer, (b) a $\mathrm{CuPc}(5 \mathrm{~nm}) / \mathrm{F}_{16} \mathrm{CuPc}(15 \mathrm{~nm})$ organic bilayer, respectively

薄膜的结晶性起到了良好的诱导作用(表1, 图2) ${ }^{[19]}$, 器 件的制备过程结合了溶液法和真空蒸镀两种方法. $\alpha-6 \mathrm{~T}$ $(\mathbf{1 A}$, 底层)/PDI-8CN2 (1E, 上层)有机双层场效应晶体 管的电子和空穴迁移率分别为 0.0035 和 $0.0095 \mathrm{~cm}^{2}$ $\mathrm{V}^{-1} \cdot \mathrm{s}^{-1}$ (表1, 图2), 实验中发现颠倒两种分子薄膜的沉
积顺序，所制备的有机双层场晶体管没有场效应性 能 ${ }^{[20]}$. 在研究有机双层2,5-双(4-联苯基)-双噻吩(BP2T) $\left(1 \mathrm{G}\right.$, 底层) $/ \mathrm{F}_{16} \mathrm{CuPc}(1 \mathrm{D}$, 上层)双极性场效应晶体管(表 1 , 图4)时发现, $\mathrm{F}_{16} \mathrm{CuPc}$ 的厚度保持不变, BP2T为5 $\mathrm{nm}$ 时该双层有机场效应晶体管的空穴/电子迁移率最高, 
分别是 $0.12,3 \times 10^{-2} \mathrm{~cm}^{2} \cdot \mathrm{V}^{-1} \cdot \mathrm{s}^{-1}, \mathrm{BP} 2 \mathrm{~T}$ 的厚度增大或 减小都会降低该有机双层晶体管的场效应性能. 原子力 显微镜下发现, 低于 $5 \mathrm{~nm}$ 的 BP2T薄膜不连续, 超过 5 $\mathrm{nm}$ 的BP2T薄膜表面粗糙度随膜厚度增加而显著增加, 5 $\mathrm{nm}$ BP2T薄膜是该双层有机场效应晶体管的最佳厚度 (图4) ${ }^{[7 \mathrm{a}]}$. 在报道的基于 $\alpha-6 \mathrm{~T}(\mathbf{1 A}$, 底层 $) / \mathrm{C}_{60}$ (1B，上层) 的双极性有机双层晶体管(表1, 图2), 所获得的空穴迁 移率和电子迁移率分别为 0.004 和 $0.005 \mathrm{~cm}^{2} \cdot \mathrm{V}^{-1} \cdot \mathrm{s}^{-1}[6]$, 该器件中两种有机小分子的化学结构相差很大, 但是获 得的两种载流子迁移率在同一个数量级, 该器件是首例 报道基于有机双层的双极性晶体管，引发了有机双极性 晶体管研究的新思路. 该报道还指出当两种材料的沉积 顺序相反时, 所获得电子迁移率为 $0.09 \mathrm{~cm}^{2} \cdot \mathrm{V}^{-1} \cdot \mathrm{s}^{-1}$, 但
空穴迁移率非常低.

此外，有机双层晶体管中 $\mathrm{p}$ 型小分子的 HOMO 能级 和 $\mathrm{n}$ 型小分子的 LUMO 能级差越小越好. 在用同一种金 属作电极时, 能级差越小, 器件的接触电阻就越小, 有 利于高性能晶体管器件的制备. 目前大多数有机半导体 的 HOMO 能级低于 $-5.20 \mathrm{eV}$, 而大多数有机半导体的 LUMO 能级高于 $-4.20 \mathrm{eV}$ (表 1)。已报道的有机双层晶 体管中大多数的两种小分子的能极差大于 $1.0 \mathrm{eV}$. 提高 $\mathrm{p}$ 型小分子的 HOMO 能级且降低小分子的 LUMO 能级, 使得两种小分子的能极差小于 $1 \mathrm{eV}$ 是今后提高有机双 层晶体管性能的有益思路. 综上所述，具有相近的共轭 骨架且轨道能级差较小的两种小分子，是有机双层晶体 管同时呈现高且匹配的空穴迁移率和电子迁移率的关 键条件.
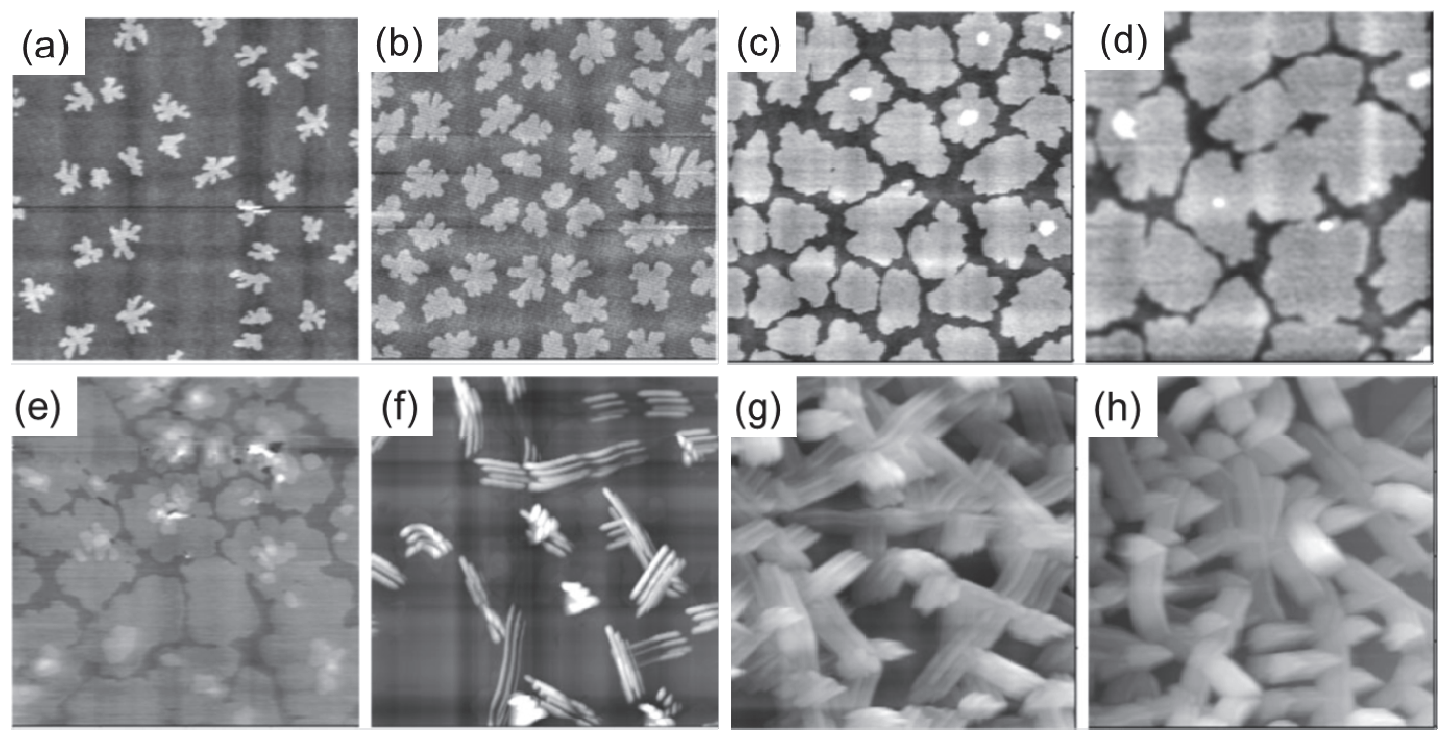

图 4 BP2T 不同厚度薄膜表面的原子力表征图

Figure 4 AFM images of BP2T films with different thicknesses

(a) $0.5 \mathrm{~nm}$, (b) $1 \mathrm{~nm}$, (c) $2 \mathrm{~nm}$, (d) $3 \mathrm{~nm}$, (e) $5 \mathrm{~nm}$, (f) $6 \mathrm{~nm}$, (g) $20 \mathrm{~nm}$, (h) $30 \mathrm{~nm}$ (all images are $4 \mu \mathrm{m} \times 4 \mu \mathrm{m}$ ) [7a] $^{2}$

表 1 基于有机一有机双层的双极性场效应晶体管的空穴迁移率、电子迁移率和 HOMO/LUMO 的总结

Table 1 Summary of hole/electron mobility, HOMO, and LUMO obtained from ambipolar OFETs based on organic-organic bilayer

\begin{tabular}{|c|c|c|c|c|}
\hline OSC & $\mu_{\mathrm{h}} /\left(\mathrm{cm}^{2} \cdot \mathrm{V}^{-1} \cdot \mathrm{s}^{-1}\right)$ & $\mu_{\mathrm{e}} /\left(\mathrm{cm}^{2} \cdot \mathrm{V}^{-1} \cdot \mathrm{s}^{-1}\right)$ & $\mathrm{HOMO} / \mathrm{eV}$ & $\mathrm{LUMO} / \mathrm{eV}$ \\
\hline $\mathbf{1 A} / \mathbf{1} \mathbf{B}^{[6]}$ & 0.004 & 0.005 & $-/-7.1$ & $-/-3.8$ \\
\hline $\mathbf{1 D} / \mathbf{1} \mathbf{C}^{[12]}$ & $2.96 \times 10^{-3}$ & $9.49 \times 10^{-3}$ & $-6.1 /-5.95$ & $-4.9 /-4.35$ \\
\hline $\mathbf{1 A} / \mathbf{1} \mathbf{E}^{[20]}$ & 0.0035 & 0.0095 & $-/-6.7$ & $-/-4.3$ \\
\hline $\mathbf{1 F} / \mathbf{1} \mathbf{D}^{[19 a]}$ & 23.7 & 0.06 & $-5.4 /-6.1$ & $-2.4 /-4.7$ \\
\hline $\mathbf{1 B} / \mathbf{1} \mathbf{H}^{[18]}$ & 0.2 & 0.04 & $-7.1 /-5.0$ & $-3.8 /-3.0$ \\
\hline $\mathbf{1 G} / \mathbf{1} \mathbf{D}^{[\mathrm{aa}]}$ & 0.12 & $3 \times 10^{-2}$ & $-/-6.1$ & $-/-4.9$ \\
\hline $\mathbf{1 D} / \mathbf{1} \mathrm{C}^{[9 \mathrm{c}]}$ & $10^{-6}$ & $10^{-6}$ & $-6.1 /-5.2$ & $-4.9 /-3.5$ \\
\hline $\mathbf{1 H} / \mathbf{1} \mathbf{I}^{[13,21]}$ & 0.41 & 0.40 & $-5.0 /-5.4$ & $-3.0 /-3.4$ \\
\hline $\mathbf{1 C} / \mathbf{1} \mathbf{J}^{[10]}$ & $1.8 \times 10^{-4}$ & $2.1 \times 10^{-4}$ & $-5.2 /-5.3$ & $-3.5 /-4.0$ \\
\hline $\mathbf{1 H} / \mathbf{1} \mathbf{K}^{[15]}$ & 0.34 & 0.03 & $-5.0 /-6.5$ & $-3.0 /-4.0$ \\
\hline $\mathbf{1 L} / \mathbf{1} \mathbf{M}^{[16]}$ & $5.0 \times 10^{-2}$ & $1.6 \times 10^{-2}$ & $-5.3 /-4.8$ & $-3.2 /-2.8$ \\
\hline $\mathbf{1 N} / \mathbf{1 O}^{[17]}$ & $1.7 \times 10^{-2}$ & $2.2 \times 10^{-2}$ & $-/-7.24$ & $-/-3.61$ \\
\hline $\mathbf{1 P} / \mathbf{1} \mathbf{Q}^{[14]}$ & 0.87 & 0.82 & $-5.75 /-5.60$ & $-4.15 /-2.60$ \\
\hline
\end{tabular}




\section{2 基于有机/无机双层的双极性场效应晶体管}

单极性有机场效应晶体管的电子传输通常比空穴

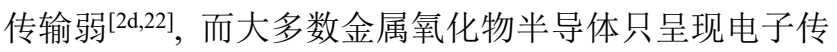
输且稳定性好 [23]. 因此, 用有机半导体作为空穴传输 层, 金属氧化物作为电子传输层, 有希望制备出高性能 且两种载流子迁移率相当的具有双层导电沟道结构的 双极性场效应晶体管，在有机复合电路的应用中有巨大 的潜力。

在这种有机一无机杂化的双极性场效应晶体管中, 并五苯 $(1 \mathrm{H}$, 图 2)一般用作 $\mathrm{p}$ 型传输层, 其空穴迁移率 与非晶硅相近 ${ }^{[24]}$. 已报道的并五苯/氧化锌双极性有机 场效应晶体管中, 器件结构为硅/二氧化硅/氧化锌 $(\mathrm{n}$ 型)/并五苯( $\mathrm{p}$ 型)/ $\mathrm{Au}$, 获得的空穴迁移率和电子迁移率 相当, 分别为 $6.3 \times 10^{-3}$ 和 $7.6 \times 10^{-3} \mathrm{~cm}^{2} \cdot \mathrm{V}^{-1} \cdot \mathrm{s}^{-1}$ (表 $2)^{[25]}$. 在并五苯和氧化锌之间的界面插入十二酸自组装 单层, 优化了并五苯薄膜中的分子排列, 使得双极性晶 体管的迁移率显著提高, 获得的空穴迁移率和电子迁移 率相当, 分别为 0.34 和 $0.38 \mathrm{~cm}^{2} \cdot \mathrm{V}^{-1} \cdot \mathrm{s}^{-1}$ [26], 电流开关 比 $\left(I_{\mathrm{on} / \mathrm{off}}\right)$ 分别为 $10^{3}$ 和 $10^{4}$. 基于该并五苯/氧化锌双极性 晶体管制备的逆变器在输入电压为 $40 \mathrm{~V}$ 时, 获得的最 大电压增益是 18.6 , 远高于基于并五苯晶体管制备的复 合电路 ${ }^{[27]}$. 用氧化铟锌代替氧化锌, 双极性传输变得不 平衡，空穴迁移率和电子迁移率分别为 0.14 和 13.8 $\mathrm{cm}^{2} \cdot \mathrm{V}^{-1} \cdot \mathrm{s}^{-1}[28]$. 用氧化铟代替氧化锌, 晶体管结构为 $\mathrm{Al}: \mathrm{Nd} / \mathrm{AlO}_{x}: \mathrm{Nd} / \mathrm{InO}_{x}$ (n 型)/聚(全氟丁烯基乙烯基醚, Cytop)/并五苯 (p 型) $/ \mathrm{Au}$, 其中聚合物作为界面修饰层, 获得的空穴迁移率和电子迁移率分别为 1.1 和 $0.1 \mathrm{~cm}^{2}$ -
$\mathrm{V}^{-1} \cdot \mathrm{s}^{-1}$ (表 2) ${ }^{[29]}$. 由该双极性晶体管制备的逆变器在输 入电压为 $50 \mathrm{~V}$ 时, 最大电压增益达到 58 , 显著高于由 $\mathrm{n}$ 型或者 $\mathrm{p}$ 型晶体管制备的逆变器 ${ }^{[30]}$

除了并五苯之外，红荧烯(2A，图 5)也被用作有机一 无机双极性场效应晶体管中的 $\mathrm{p}$ 型有机半导体层. 晶体 管结构为硅/二氧化硅/二硫化钼( $\mathrm{n}$ 型)/红荧烯( $\mathrm{p}$ 型)/ $\mathrm{Au}$, 获得的电子和空穴迁移率分别为 1.27 和 $0.36 \mathrm{~cm}^{2} \bullet \mathrm{V}^{-1}$. $\mathrm{s}^{-1}$ (表 2) ${ }^{[31]}$, 两种类型载流子的开关比都为 $10^{3}$. 有机半 导体为 2,8-二氟-5,11-双 (三乙基甲硅烷基乙炔基)二噻吩 葱(diF-TESADT) (2B，图 5)和金属氧化物为氧化锌时, 双极性晶体管获得了非常高的空穴 $\left(2.4 \mathrm{~cm}^{2} \cdot \mathrm{V}^{-1} \cdot \mathrm{s}^{-1}\right)$ 和 电子迁移率为 $\left(4.5 \mathrm{~cm}^{2} \cdot \mathrm{V}^{-1} \cdot \mathrm{s}^{-1}\right)^{[32]}$, 电流开关比为 $10^{3}$. 基于该双极性晶体管制备的逆变器在输入电压为 $60 \mathrm{~V}$ 时, 获得的电压增益超过 20. 有机半导体为 C8-BTBT (2C，图 5) 和金属氧化物为氧化铟镓锌(a-IGZOs)时，双 极性晶体管获得了非常高的空穴迁移率 $\left(4.5 \mathrm{~cm}^{2} \cdot \mathrm{V}^{-1}\right.$ ・ $\left.\mathrm{s}^{-1}\right)$ 和电子迁移率为 $\left(5.1 \mathrm{~cm}^{2} \cdot \mathrm{V}^{-1} \cdot \mathrm{s}^{-1}\right)$. 有机半导体为 C10-DNTT (1F) 和金属氧化物为氧化铟镓锌 (a-IGZOs) 时, 双极性晶体管也获得了比较高的空穴 $\left(2.8 \mathrm{~cm}^{2} \cdot \mathrm{V}^{-1}\right.$ ・ $\left.\mathrm{s}^{-1}\right)$ 和电子迁移率为 $\left(5.1 \mathrm{~cm}^{2} \cdot \mathrm{V}^{-} 1 \cdot \mathrm{s}^{-1}\right)^{[33]}$. 基于该双极 性晶体管制备的逆变器在输入电压为 $60 \mathrm{~V}$ 时, 获得的 电压增益高达 124(图 6).

在有机一无机双层有机场效应晶体管中，聚合物半 导体也被成功应用于 $\mathrm{p}$ 型传输层. 例如，基于聚(3-已基 噻吩)(1N, $\mathrm{p}$ 型)和氧化锌( $\mathrm{n}$ 型)制备的晶体管呈现了平衡 的空穴迁移率和电子迁移率, 分别为 $1.2 \times 10^{-2}, 5.1 \times$ $10^{-2} \mathrm{~cm}^{2} \cdot \mathrm{V}^{-1} \cdot \mathrm{s}^{-1}[34]$. 基于聚(3-已基噻吩)(1N, $\mathrm{p}$ 型)和

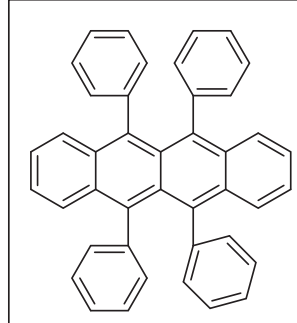

2A

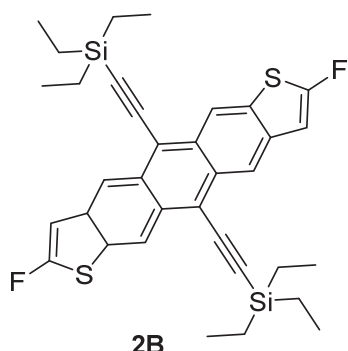

2B

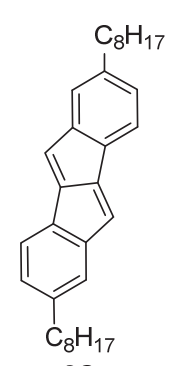

2C

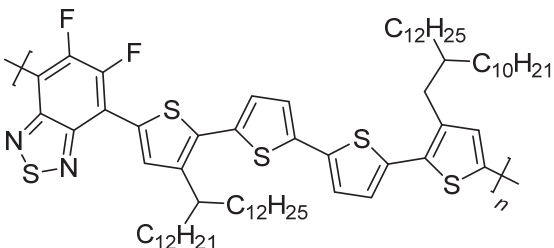

2D

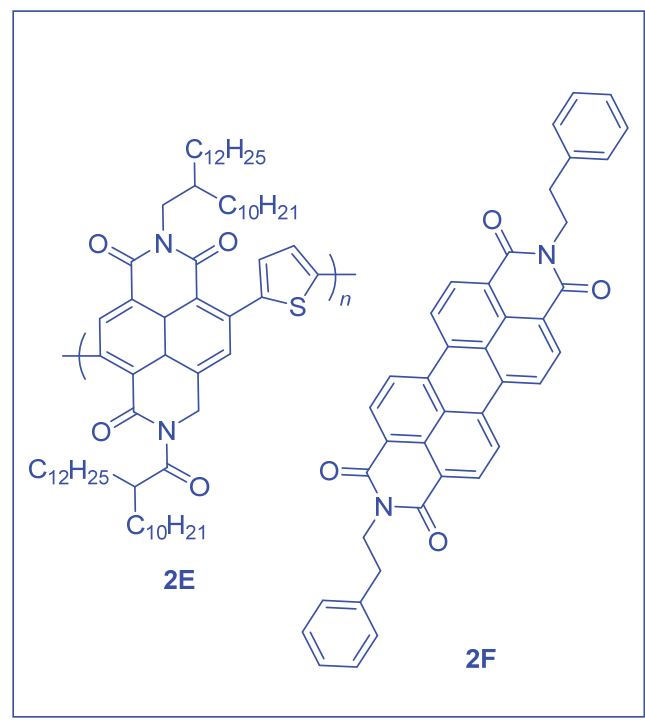

n-type OSCs

p-type OSCs

图 5 双极性场效应晶体管中有机一无机双层及平行导电沟道的有机半导体分子结构式

Figure 5 Molecular structures of OSCs applied in the ambipolar OFETs based on organic-inorganic bilayer and parallel conducting channel 
氧化铟镓( $\mathrm{n}$ 型)制备的晶体管呈现了平衡的且较高的空 穴迁移率和电子迁移率, 分别为 $1.09,1.80 \mathrm{~cm}^{2} \cdot \mathrm{V}^{-1}$ ・ $\mathrm{s}^{-1[\mathbf{B}]}$, 对应的电流开关比分别为 $10^{2}$ 和 $10^{3}$. 基于该双 极性晶体管制备的逆变器在输入电压为 $-2 \mathrm{~V}$ 时, 获得 的电压增益约为 2. FBT-Th4 是一种给体-受体(D-A)型 的低带隙共轭聚合物, 将 FBT-Th4 (2D, p 型) 和氧化铟( $(n$ 型)作为晶体管的导电沟道层, 制备的晶体管展现出了 1.1 和 $1.5 \mathrm{~cm}^{2} \cdot \mathrm{V}^{-1} \cdot \mathrm{s}^{-1}$ 的空穴迁移率和电子迁移率 ${ }^{[35]}$, 两种载流子的迁移率都比较高且相当, 电流开关比都为 $10^{2}$.

如表 2 所示, 有机/无机双层晶体管中, $\mathrm{p}$ 型有机分 子的 HOMO 能级和 $\mathrm{n}$ 型无机金属氧化物的导带能级差 比较小, 一般低于 $1.0 \mathrm{eV}$, 这应该是有机/无机双层晶体 管迁移率显著高于有机双层晶体管迁移率的一个主要 原因. 多个有机/无机双层晶体管的空穴迁移率和电子 迁移率都大于 $1.0 \mathrm{~cm}^{2} \cdot \mathrm{V}^{-1} \cdot \mathrm{s}^{-1}$, 与无定形硅的迁移率相 当. 在有机/无机双层晶体管中, 一般先制备无机金属氧 化物薄膜, 后制备有机半导体薄膜. 这是因为金属氧化
物的制备一般是通过高温溅射法或者先旋涂再高温煅 烧的方法, 这两种方法的操作温度都很高, 会破坏有机 分子的化学结构. 此外, 金属氧化物薄膜表面的粗粘度 比较低, 为 $0.1 \mathrm{~nm}$ 左右, 与硅片表面粗粘度相当, 能更 好的诱导上层有机薄膜的有序生长. 综上所述，基于有 机/无机双层的双极性场效应晶体管可同时获得比较高 的且相当的电子迁移率和空穴迁移率, 展现出在有机互 补电路中广阔的应用前景.

基于平行 $\mathrm{p} / \mathrm{n}$ 导电沟道的双极性有机场效应晶体管 平行 $\mathrm{p}$ 沟道和 $\mathrm{n}$ 沟道有机场效应晶体管的器件结构如图 7 所示. 与有机双层器件相比, 平行导电沟道器件中不 存在有机层一有机层界面, 这个界面一般有较大的注入 势垒，会降低有机层中分子排列有序度，从而降低有机 场效应晶体管电学性能. 因此, 这种平行沟道的器件结 构在制备高性能双极性有机场效应晶体管方面显示出 较大的优势. 如真空蒸镀法制备的有机平行沟道 $\mathrm{CuPc}$ (1C)和 $\mathrm{F}_{16} \mathrm{CuPc}(1 \mathrm{D})$, 金 $(-5.1 \mathrm{eV})$ 作为源/漏电极, 其功 函与 $\mathrm{CuPc}(1 \mathrm{C},-5.0 \mathrm{eV})$ 的 $\mathrm{HOMO}$ 和 $\mathrm{F}_{16} \mathrm{CuPc}(1 \mathrm{D}$, (a)

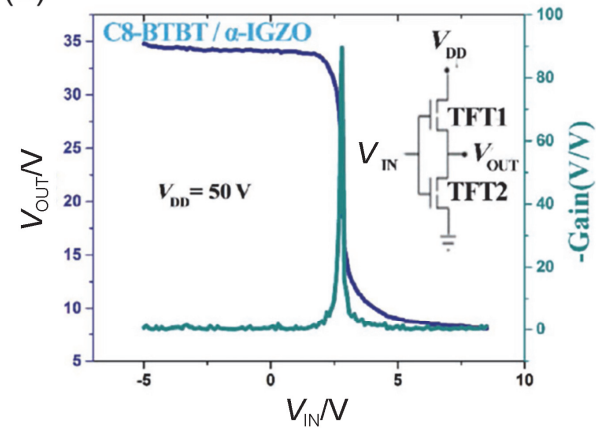

(b)

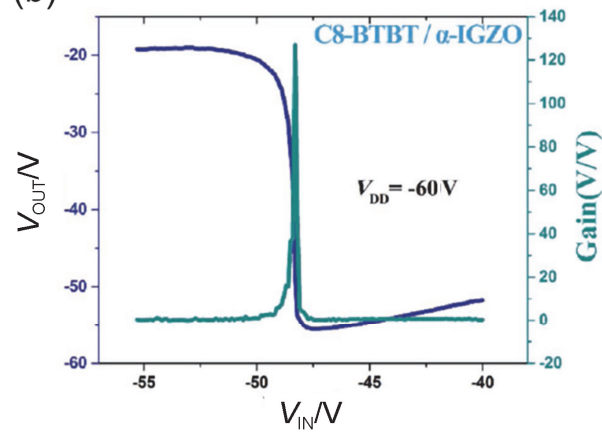

图 6 基于 C8-BTBT/a-IGZO 反相器输入电压为 $50 \mathrm{~V}$ (a) 和 $-60 \mathrm{~V}(\mathrm{~b})$ 时的转移曲线 ${ }^{[33]}$

Figure 6 Supply voltages at $50 \mathrm{~V}$ (a) and $-60 \mathrm{~V}$ (b) based on C8-BTBT/a-IGZO inverters Inset is schematic of the inverter circui

表 2 基于有机/无机双层的双极性场效应晶体管的空穴迁移率、电子迁移率、电流开关比、HOMO 和导带能级的总结

Table 2 Summary of $\mu_{\mathrm{h}}, \mu_{\mathrm{e}}, I_{\mathrm{on} / \mathrm{off}}$, HOMO, conducting band obtained from ambipolar OFETs based on organic-organic bilayer

\begin{tabular}{|c|c|c|c|c|c|c|}
\hline OSC & $\mu_{\mathrm{h}} /\left(\mathrm{cm}^{2} \cdot \mathrm{V}^{-1} \cdot \mathrm{s}^{-1}\right)$ & $I_{\mathrm{on} / \mathrm{off}(\text { hole })}$ & $\mu_{\mathrm{e}} /\left(\mathrm{cm}^{2} \cdot \mathrm{V}^{-1} \cdot \mathrm{s}^{-1}\right)$ & $I_{\text {on/off(electron) }}$ & $\mathrm{HOMO} / \mathrm{eV}$ & Conducting band/eV \\
\hline $\mathrm{ZnO} / \mathbf{1} \mathbf{H}^{[26]}$ & 0.34 & $10^{3}$ & 0.38 & $10^{4}$ & -5.0 & -4.4 \\
\hline $\mathrm{ZnInO}_{x} / \mathbf{1} \mathbf{H}^{[28]}$ & 0.14 & - & 13.8 & - & -5.0 & - \\
\hline $\operatorname{InO}_{x} / \mathbf{1} \mathbf{H}^{[29]}$ & 1.1 & - & 0.1 & - & -5.0 & - \\
\hline $\mathrm{MoS}_{2} / \mathbf{2} \mathbf{A}^{[31]}$ & 0.36 & $10^{3}$ & 1.27 & $10^{3}$ & -5.36 & -4.0 \\
\hline $\mathrm{ZnO} / \mathbf{2} \mathbf{B}^{[32]}$ & 2.4 & $10^{3}$ & 4.5 & $10^{3}$ & - & -4.4 \\
\hline IGZOs/2C ${ }^{[33]}$ & 4.5 & - & 5.1 & - & -5.3 & -4.3 \\
\hline IGZOs/1F $\mathbf{F}^{[33]}$ & 2.8 & - & 5.1 & - & -5.1 & -4.3 \\
\hline $\mathrm{ZnO} / \mathbf{1} \mathbf{N}^{[34]}$ & $5.1 \times 10^{-2}$ & - & $1.2 \times 10^{-2}$ & - & -5.1 & -4.3 \\
\hline $\mathrm{IGO} / \mathbf{1} \mathbf{N}^{[\mathbf{1 B}]}$ & 1.09 & $10^{2}$ & 1.80 & $10^{3}$ & - & - \\
\hline $\mathrm{InO} / \mathbf{2} \mathrm{D}^{[35]}$ & 1.1 & $3.8 \times 10^{2}$ & 1.5 & $1.2 \times 10^{2}$ & -5.36 & - \\
\hline
\end{tabular}


(a)

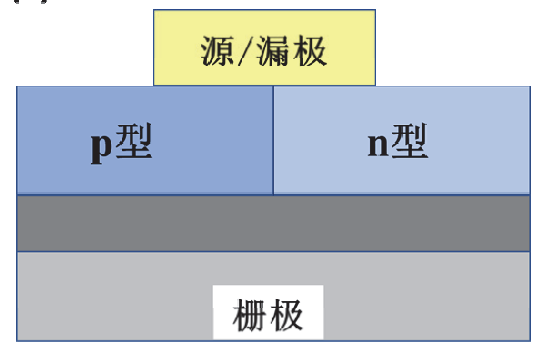

(b)

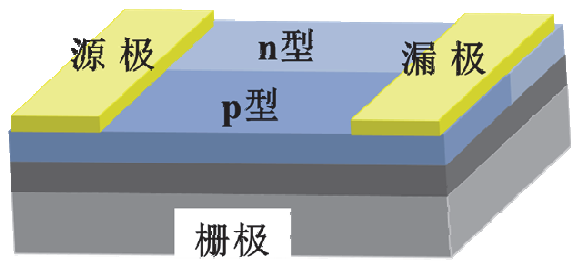

图 7 基于平行导电沟道的双极性有机场效应晶体管: (a)正面示意图和(b)侧面示意图

Figure 7 Device structure of ambipolar OFETs with parallel $\mathrm{p}$ and $\mathrm{n}$ conducting channel: (a) front view and (b) side view

$-4.8 \mathrm{eV})$ 的 LUMO 比较接近 ${ }^{[36]}$, 获得的空穴迁移率和 电子迁移率分别为 $1.40 \times 10^{-2}$ 和 $8.69 \times 10^{-3} \mathrm{~cm}^{2} \cdot \mathrm{V}^{-1}$ ・ $\mathrm{s}^{-1}$. 平行沟道为 $\mathrm{CuPc}$ 和 $\mathrm{F}_{16} \mathrm{CuPc}$ 的微纳晶时, 载流子传 输性能明显提高且两种载流子迁移率相当, 空穴迁移率 和电子迁移率分别为 0.1 和 $0.17 \mathrm{~cm}^{2} \cdot \mathrm{V}^{-1} \cdot \mathrm{s}^{-1}$ [37]. 基于 平行 P3HT (1N) 和 P(NDI-T) (2E, 图 5)纳米线的双极性 有机场效应晶体管呈现出平衡的空穴迁移率和电子迁 移率，即 $8.2 \times 10^{-2} / 7.5 \times 10^{-2} \mathrm{~cm}^{2} \cdot \mathrm{V}^{-1} \cdot \mathrm{s}^{-1}$ [38]. 用提前 刻蚀好的带状光刻胶作为模板, 诱导 $\mathrm{p}$ 型单晶 diF-TESADT (2B, 图 5)的生长, 再以 $\mathrm{p}$ 型单晶为模板, 诱导 $\mathrm{n}$ 型单晶 BPE-PTCDI (2F, 图 5)的单晶生长, 该方 法制备了大面积平行的行 $\mathrm{p}$ 型和 $\mathrm{n}$ 型导电沟道, 制备的 双极性器件的空穴迁移率和电子迁移率为 0.32 和 0.43 $\mathrm{cm}^{2} \cdot \mathrm{V}^{-1} \cdot \mathrm{s}^{-1}$ [39]. 平行导电沟道晶体管的电学性能比有 机双层导电沟道的晶体管有优势, 但是平行导电沟道中 两种有机薄膜的沉积过程比较繁琐且成本高, 因为在沉 积第二种有机薄膜时需要用掩膜版遮掩第一种有机薄 膜, 蒸镀源漏电极之前还需要拆除有机薄膜上的模板.

\section{4 基于 $p / n$ 半导体混合物的双极性有机场效应晶体 管}

基于有机双层的双极性晶体管在同时获得高电子 和高空穴迁移率方面取得了显著效果. 但是大多数有机 双层晶体管只能通过真空沉积方法来制备, 成本比较 高. 如果用成本低的溶液法制备有机薄膜, 先沉积的底 层薄膜会被后沉积的上层薄膜溶解, 不利于高性能双极 性器件的制备. 使用 $\mathrm{p} / \mathrm{n}$ 两种类型半导体的混合物可以 直接使用溶液法制备有机薄膜, 实现双极性电荷传输. 因此, 基于 $\mathrm{p} / \mathrm{n}$ 有机半导体混合物的方法制备双极性有 机晶体管，能显著降低成本，在实际应用中更有优势.

已报道的基于有机半导体混合物的双极性晶体管 中通常含有一种 $\mathrm{n}$ 型半导体和一种 $\mathrm{p}$ 型半导体. 其中 $\mathrm{p}$ 型半导体 P3HT (1N) 经常用于 $\mathrm{p} / \mathrm{n}$ 半导体混和物薄膜中, 如图 2 和图 8 中混合物 P3HT (1N)/PC ${ }_{71 \mathbf{B M}}(\mathbf{8 A})^{[40] 、}$ P3HT (1N)/P(NDI-T) (8B) $)^{[38]} 、$ P3HT (1N)/P(NDI2OD-T2) $(\mathbf{8 C})^{[41]} \cdot \mathrm{n}$ 型富勒烯 $(\mathbf{8 A})$ 也经常与 $\mathrm{DPP}(\mathrm{TBFu})_{2}(\mathbf{8 D})^{[40]}$, OC1C10-PPV (8E) $)^{[42]}$ 等 $\mathrm{p}$ 型半导体混合(图 8). 由于混合 物薄膜中两种分子相互干扰, 分子堆积的有序性一般比 较差, 获得的载流子迁移率一般不高于 $10^{-2} \mathrm{~cm}^{2} \cdot \mathrm{V}^{-1}$ • $\mathrm{s}^{-1}$. 而且, 混合物薄膜中载流子的迁移率通常低于在单 个组分薄膜中的迁移率 [43]. 即使对于全共轭半导体并 五苯(1H)/PTCDI-C13 (1I)混和物薄膜中的空穴迁移率 也仅为 $0.09 \mathrm{~cm}^{2} \cdot \mathrm{V}^{-1} \cdot \mathrm{s}^{-1}$, 比单组份并五苯的迁移率低 一个数量级.

总而言之，基于有机双层的双极性晶体管的载流子 迁移率一般高于基于 $\mathrm{p} / \mathrm{n}$ 有机混合物晶体管的载流子迁 移率. 但是有机双层晶体管需要真空蒸镀法制备，该方 法制备成本高, 不利于工业应用; 而基于 $\mathrm{p} / \mathrm{n}$ 有机混合 物的晶体管可用溶液法制备，该方法制备成本低，更适 合于工业应用. 有机双层晶体管和有机混合物晶体管各 有优缺点，应用中要按照实际情况进行选择.

\section{2 影响双极性晶体管电学性能的因素}

\section{1 金属电极}

一般来说, 有机半导体的 $\mathrm{HOMO}$ 能级为 $-4.9 \sim$ $-5.5 \mathrm{eV}, \mathrm{LUMO}$ 能级为 $-3.0 \sim-4.0 \mathrm{eV}$. 所有已报道的 有机半导体分子的 HOMO 和 LUMO 之间都存在带隙, 且大多数有机半导体分子的带隙大于 $2.0 \mathrm{eV}$. 在有机双 层场效应晶体管中, $\mathrm{p}$ 型半导体的 HOMO 能级和 $\mathrm{n}$ 型半 导体的 LUMO 能级也存在带隙. 当施加一个金属电极 时, 至少有一种类型载流子的传输会受到注入势垒的限 制. 选择功函匹配的金属电极可以降低注入势垒，增加 晶体管中载流子的注入效率，提高晶体管的电学性能. 为提高注入效率, 有机半导体分子的 HOMO 和 LUMO 应同时与金属电极的功函相匹配. 功函比较高的电极如 金 $(-5.1 \mathrm{eV})$, 与 HOMO 能级更匹配, 适合空穴注入 ${ }^{[44]}$. 有机电极四氰基二甲烷四硫富瓦烯(TTF-TCNQ)的功函 为 $-4.64 \sim-4.78 \mathrm{eV}$, 也更适合做空穴注入电极 ${ }^{[45]}$. 低 功函金属如 $\mathrm{Ag}(-4.26 \mathrm{eV}) 、 \mathrm{Al}(-4.28 \mathrm{eV}) 、 \mathrm{Mg}(-3.66$ 
$\mathrm{eV})$ 和 $\mathrm{Ca}(-2.87 \mathrm{eV})$ 等能显著改善电子注入 ${ }^{[44-45]}$. 因此 使用不对称电极是降低双极性晶体管中注入势垒的有 效方法, 即在双极性晶体管中同时使用一个高功函电极 用于空穴注入和一个低功函电极用于电子注入, 该方法 能很好地实现空穴和电子传输的平衡 ${ }^{[46]}$.

\section{2 电极和有机半导体层之间的缓冲层}

在已报道的有机半导体材料中, 电子迁移率通常低 于空穴迁移率 ${ }^{[47]}$. 在金属电极和有机半导体层之间插 入一层缓冲层, 是提高电子迁移率的有效办法. 缓冲层 的主要作用是降低金属的功函, 能更好地与有机半导体 材料的 LUMO 能级相匹配. 电极缓冲层可以调控金属 电极的功函. 如金属 Mo $(-4.5 \mathrm{eV})$ 的功函可通过加入 $\mathrm{V}_{2} \mathrm{O}_{5}$ 和聚乙烯酰亚胺 (PEI) 的混合物来调控. 当混合比 例为 $7: 3$ 时, Mo 的功函下降到 $-4.32 \mathrm{eV}$, 与吡咯并吡 咯二酮和噻吩的聚合物(DPPT-TT)的 LUMO 能级匹配, 电子迁移率从 $0.14 \mathrm{~cm}^{2} \bullet \mathrm{V}^{-1} \cdot \mathrm{s}^{-1}$ 提高到 $0.6 \mathrm{~cm}^{2} \cdot \mathrm{V}^{-1} \cdot \mathrm{s}^{-1}$, 电子传输性能得到显著改善 ${ }^{[48]}$. 在反向印刷制备的银 电极上插入碳酸铯, 功函从 -4.96 降为 $-4.5 \mathrm{eV}$, 使吡 咯并吡咯二酮和噻吩的共聚物(PDPP-TAT)电子迁移率 提高了近一个数量级 ${ }^{[49]} . \mathrm{CsF}$ 可以用来调控 $\mathrm{Au}$ 电极的功 函 ${ }^{[50]}$. 在金属电极和半导体之间添加一层无机盐薄膜, 可以防止金属在蒸镀过程中扩散到有机半导体薄膜中, 或防止半导体和金属之间发生有机化学反应. 对于底接 触结构的晶体管, 在电极表面修饰一层自组装单分子
膜，也可以改变金属表面的功函，从而改善有机半导体 材料的载流子注入. 例如, 用五氟苯硫醇(PFBT)或 $1 H, 1 H, 2 H, 2 H$ - 全氟十二烷硫醇 (PFDT) 和 1 - 癸硫醇 (1-DT)的混合物进行修饰，能显著降低金电极表面的功 函 ${ }^{[51]}$.

\section{3 介电层}

场效应晶体管中导电沟道位于介电层上方的有机 半导体层 1 2 nm 内, 因此介电层的表面性质极易影响 有机半导体薄膜中的分子排列, 对晶体管的电学性能影 响比较大. 二氧化硅是一种常用的介电层, 表面有许多 $\mathrm{OH}$ 基团, 亲水性比较强, 不利于疏水性有机半导体分 子在二氧化硅表面的有序排列, 而在二氧化硅表面修饰 一层硅烷可以消除 $\mathrm{OH}$ 基团, 大大降低陷阱密度 ${ }^{[52]}$. 绝 缘聚合物也可以用作介电层 ${ }^{[8 a]}$, 聚合物薄膜的粗糙度、 介电常数 $(k)$ 和界面偶极子等因素会影响载流子传输. 绝缘聚合物薄膜通常用旋涂法沉积在栅极表面，其表面 的粗楉度应控制在 $0.5 \mathrm{~nm}$ 以内, 这与二氧化硅表面粗粘 度一致. 聚合物薄膜表面粗䊁度太大, 会阻碍有机半导 体分子在介电层上的有序排列, 有机晶粒不能长大, 晶 体管的电学性能比较低 ${ }^{[44 c]}$. 绝缘聚合物的介电常数也 会影响晶体管的性能,一般低介电常数的绝缘聚合物的 场效应性能明显优于高介电常数绝缘聚合物 ${ }^{[4 c]}$. 如基 于四正已基双二氧基乙烯联四噻吩 $\left[\mathrm{QQT}(\mathrm{CN})_{4}\right]$ 的晶体 管用 AL-X501 $(k=2.8)$ 为介电层时, 空穴/电子迁移率分

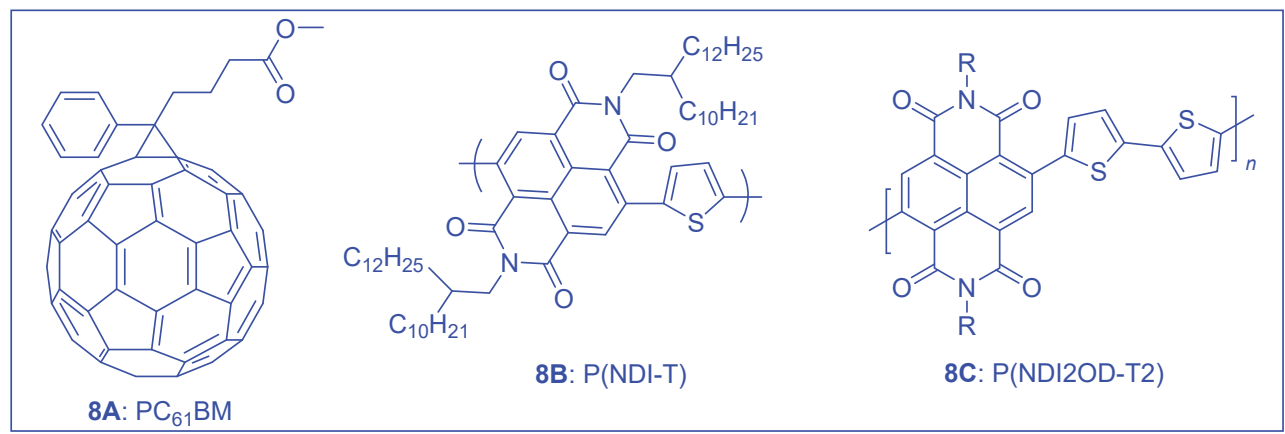

n-type OSCs

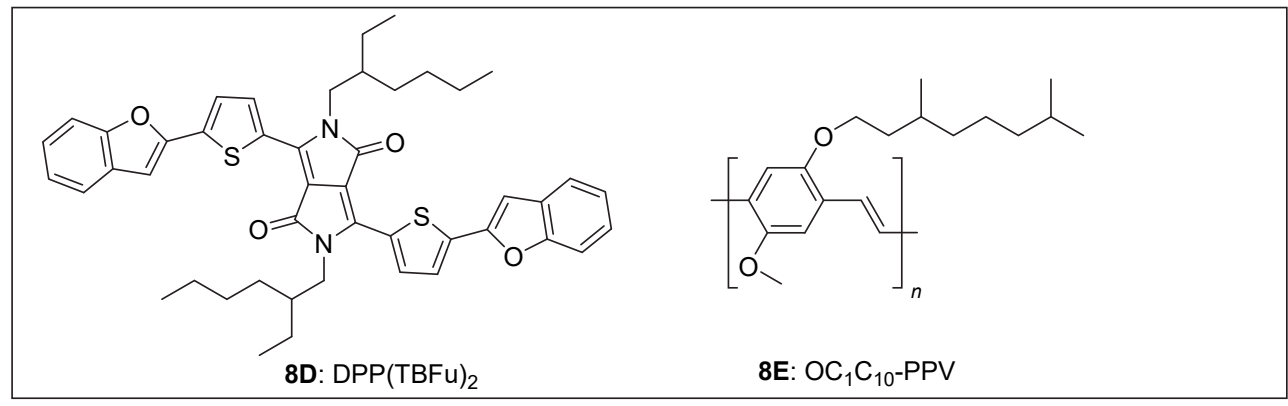

p-type OSCs

图 8 双极性有机场效应晶体管导电沟道为 $\mathrm{p} / \mathrm{n}$ 有机半导体混合物的分子结构式

Figure 8 Molecular structures of OSCs applied in the ambipolar OFETs based on $\mathrm{p} / \mathrm{n}$ organic blends 
别是 0.04 和 $0.007 \mathrm{~cm}^{2} \cdot \mathrm{V}^{-1} \cdot \mathrm{s}^{-1}$, 是用聚乙烯基苯酚 (PVP, $k=3.6$ ) 和聚酰亚胺(PI, $k=3.8$ ) 作为介电层的两倍. 高介电常数的绝缘聚合物通常存在随机排列的偶极子, 增加了介电层表面的缺陷密度, 降低了晶体管的载流子 传输, 这种现象对非晶态有机半导体材料影响比较明 显. 而结晶度高和侧链较长的有机半导体材料几乎不受 影响. 高介电常数的介电层可以降低器件的工作电压和 能耗, 有利于在实际应用中实现绿色节能. 如以高介电 常数化合物聚偏氟乙烯三氟乙烯 $(\mathrm{P}(\mathrm{VDF}-\mathrm{TrFE}))$ 为介电 层, 基于菜酰亚胺和噻吩的聚合物(P(NDI2OD-T2))在操 作电压仅为 $30 \mathrm{~V}$ 时就可以正常工作, 获得的空穴迁移 率和电子迁移率分别是 0.11 和 $0.093 \mathrm{~cm}^{2} \bullet \mathrm{V}^{-1} \bullet \mathrm{s}^{-1}[53]$. 实验研究发现, 介电层 $\mathrm{P}(\mathrm{VDF}-\mathrm{TrFE})$ 中的 C-F 强偶极子 增强了正电荷载流子在半导体层一介电层界面的积累, 使得聚合物 P(NDI2OD-T2)的 HOMO/LUMO 能级升高 (图9), 注入势垒变得降低, 因而晶体管的电学性能得到 提高 ${ }^{[54]}$.

\section{5 有机半导体层的沉积顺序}

有机半导体材料的沉积顺序也是影响有机双层双 极性场效应晶体管电学性能的重要因素. 沉积顺序决定 了有机双层界面的形貌, 界面越光滑, 双极性传输性能 越好. 有机双层的界面受底层有机薄膜表面形貌的影响 比较大. 如有机半导体 $\alpha-6 \mathrm{~T}$ (1A) 先沉积时, 有机双层 $1 \mathrm{~A} / 1 \mathrm{~B}$ 场效应晶体管的空穴迁移率和电子迁移率分别为 0.004 和 $0.005 \mathrm{~cm}^{2} \cdot \mathrm{V}^{-1} \cdot \mathrm{s}^{-1}[6]$. 而如果先沉积有机半导体 $\mathrm{C}_{60}(\mathbf{1 B})$, 有机双层 $1 \mathrm{~B} / \mathbf{1 A}$ 晶体管只能测出 $\mathrm{n}$ 型传输, 无 空穴传输. 实验发现 $\mathrm{C}_{60}(\mathbf{1 B})$ 薄膜的表面比 $\alpha-6 \mathrm{~T}$ (1A) 薄

\section{4 有机半导体层的厚度}

基于有机双层的双极性有机场效应晶体管的电学 性能与底层有机层的厚度有关. 底层有机层表面越粗 粘, 产生电荷散射越强, 上层有机层的缺陷密度会增加, $\mathrm{az}$ 不利于高性能晶体管器件的制备. 如 $\mathrm{CuPc}(\mathbf{1 C}, \mathrm{p}$ 型, 底)层) $/ \mathrm{SnCl}_{2} \mathrm{Pc}(\mathbf{1 J}, \mathrm{n}$ 型，上层)有机双层场效应晶体管， 分别制备了 4, 12 和 $16 \mathrm{~nm}$ 三种膜厚的底层有机薄膜 $\mathrm{CuPc}$, 并保持顶层 $\mathrm{SnCl}_{2} \mathrm{Pc}$ 膜厚为 $60 \mathrm{~nm}$ 不变, 实验发 现晶体管电子迁移率随着 $\mathrm{CuPc}$ 厚度的增加而减小 ${ }^{[10]}$. 基于有机双层并五苯(1H, $\mathrm{p}$ 型，底层)/NTFBII (1K, $\mathrm{n}$ 型, 上层)和有机双层 AC5-CF3 (1L, n 型，底层/BP3T (1M, p 型，上层)的场效应晶体管的电子迁移率也发生了类似 的实验现象 ${ }^{[55]}$. 实验中还发现当并五苯薄膜厚度小于 2 $\mathrm{nm}$ 时, 有机双层中的空穴迁移率会随着并五苯薄膜厚 度的增加而提高, 这归因于在薄膜厚度较低时并五苯薄 膜的连续性得到提高 ${ }^{[15]}$.

膜表面粗糙得多, 先沉积的 $\mathrm{C}_{60}(\mathbf{1 B})$ 薄膜严重影响 了上层 $\alpha-6 \mathrm{~T}(\mathbf{1 A})$ 薄膜的分子排列, 大大降低了晶体管 的电学性能. 有机双层 $\alpha-6 \mathrm{~T}(\mathbf{1 A}) / \mathrm{PDI}-8 \mathrm{CN} 2$ (1E) 晶体管 也展现出类似的实验现象，当有机半导体 $\alpha-6 \mathrm{~T}(\mathbf{1 A})$ 先 沉积时, 晶体管的空穴迁移率和电子迁移率分别为 0.0095 和 $0.0035 \mathrm{~cm}^{2} \cdot \mathrm{V}^{-1} \cdot \mathrm{s}^{-1}$; 而当有机半导体 PDI$8 \mathrm{CN} 2(\mathbf{1 E})$ 先沉积时晶体管的 $\mathrm{n}$ 型传输和 $\mathrm{p}$ 型传输都不 能测出 ${ }^{[20]}$.

\section{6 测试环境}

电子传输比空穴传输更易受到测试环境的影响, 是 双极性有机场效应晶体管中电子迁移率通常低于空穴 迁移率的一个重要原因 ${ }^{[55-56]}$. 空气中的水分子或氧气分 子在导电沟道中进行掺杂, 相当于增加了缺陷密度, 使 晶体管的电学性能降低 ${ }^{[55,57]}$. 在氮气、氩气氛围或手套 箱中测试器件, 能降低水分子和氧气分子密度, 更容易 获得高性能的双极性电荷传输. 如基于吡咯并吡咯二 酮、荎酰亚胺和噻吩三个单元的共聚物(PDPP2T-BT-co$\mathrm{NDI})$ 的晶体管在氩气氛围中测得的电子迁移率为 0.98 $\mathrm{cm}^{2} \cdot \mathrm{V}^{-1} \cdot \mathrm{s}^{-1}$; 但在大气环境中测得的电子迁移率仅为 $0.14 \mathrm{~cm}^{2} \cdot \mathrm{V}^{-1} \cdot \mathrm{s}^{-1[55]}$.

(a)

(b)

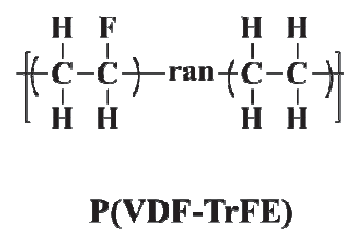

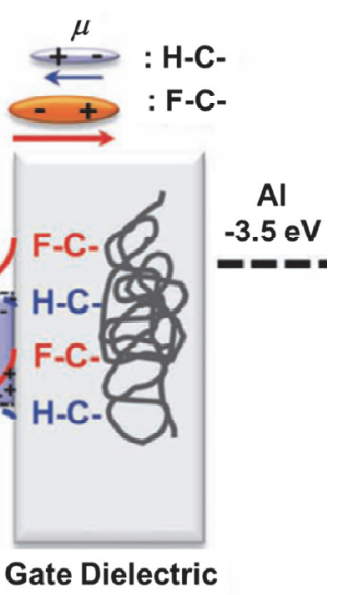

图 9 (a)介电层聚合物 $\mathrm{P}(\mathrm{VDF}-\mathrm{TrFE})$ 的化学结构式和(b)有机半导体材料和介电层界面的前沿轨道能级示意图[53]

Figure 9 (a) Molecular structure of $\mathrm{P}(\mathrm{VDF}-\mathrm{TrFE})$ and (b) schematic diagram of the energy levels at the OSCs-dielectric interface 


\section{7 器件结构}

器件结构也是影响双极性有机场效应晶体管电学 性能的一个重要因素 ${ }^{[58]}$. 如图 10 所示, 有机双层有机 场效应晶体管主要有四种器件结构, 分别是顶接触型、 底接触型、中间接触型和双栅极型. 顶部接触型由于注 入面积大，一般能获得最佳的有机场效应晶体管性 能 ${ }^{[59]}$. 底部接触型由于注入面积小, 获得的载流子迁移 率比较低, 但底部接触型有利于批量生产[59-60]. 中间接 触型包括上层有机层的底接触和底层有机层的顶接触, 可用于调节空穴迁移率和电子迁移率之间的差异, 优化 双极性有机场效应晶体管电学性能 ${ }^{[19 c, 61]}$. 在双栅极型 有机场效应晶体管中, 空穴沟道和电子沟道具有独立的 绝缘体界面, 可通过调控各自的绝缘层界面性质来调控 两种载流子的传输, 实现空穴迁移率和电子迁移率相当 的双极性有机场效应晶体管 ${ }^{[62]}$.

\section{8 其他影响因素}

其他因素如溶剂和温度也会影响器件性能. 采用旋 涂法制备有机薄膜时, 溶解有机半导体的溶剂对场效应 晶体管性能影响比较大. 如分别使用氯仿、氯苯、1,2二氯苯和 1,2,4-三氯苯制备的给体(硒吩)-受体(吡咯并 吡咯二酮)型聚合物薄膜( PSeDPP), X 射线衍射表明高沸 点溶剂 1,2-二氯苯和 1,2,4-三氯苯制备的聚合物薄膜的 衍射峰更强, 即薄膜中的分子排列更加有序. 与氯仿相 比, 由 1,2 -二氯苯和 $1,2,4$-三氯苯溶剂制成的薄膜场效 应晶体管的电子和空穴迁移率高出一个数量级 ${ }^{[63]}$. 这 是因为在旋涂制备聚合物薄膜过程中, 高沸点溶剂需要
较长的挥发时间，促进了有机膜中聚合物分子的自组 装，形成了更加有序的分子排列[64].

温度也是影响晶体管中电荷传输的一个重要因素. 对有机半导体薄膜进行热处理可以显著提高有机薄膜 的均匀性和结晶度, 从而改善晶体管的电学性能. 确定 最佳温度是热处理的关键. 有机薄膜的热处理通常需要 在真空或氮气下进行, 以避免有机半导体分子被氧化.

\section{3 结论与展望}

本综述中查阅了 118 篇文献, 涉及到 30 多个有机半 导体分子, 其中电子和空穴迁移率都大于 $1.0 \mathrm{~cm}^{2} \bullet \mathrm{V}^{-1}$. $\mathrm{s}^{-1}$ 的文献有 5 篇. 文章中按照导电沟道层的结构主要总 结了三种类型的双极性场效应晶体管, 即有机双层晶体 管、平行导电沟道晶体管和 $\mathrm{p} / \mathrm{n}$ 混合物晶体管. 从制备 工艺方面上说, $\mathrm{p} / \mathrm{n}$ 混合物晶体管最简单, 导电沟道薄膜 只需一次沉积过程; 有机双层晶体管和平行导电沟道晶 体管需要两次沉积过程, 其中平行导电沟道晶体管还需 要添加掩膜版, 制备工艺最复杂. 从制备成本方面来看, $\mathrm{p} / \mathrm{n}$ 混合物晶体管可用溶液法制备, 成本最低; 有机双 层晶体管需要真空蒸镀设备而平行导电沟道晶体管需 要掩膜版, 成本增加. 从晶体管的电学性能来看, 有机 双层晶体管和平行导电沟道晶体管的电学性能都比较 高, 而 $\mathrm{p} / \mathrm{n}$ 混合物晶体管的电学性能通常比较低. 综合 考虑，作者认为有机双层晶体管具有更大的实际应用前 景.

基于有机双层的双极性场效应晶体管是目前获得 (a)

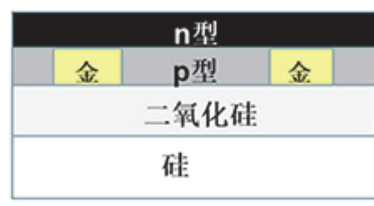

(b)

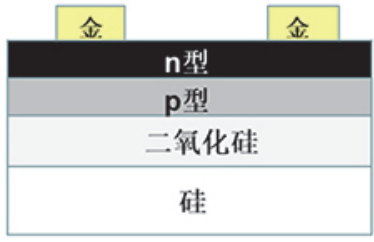

(c)

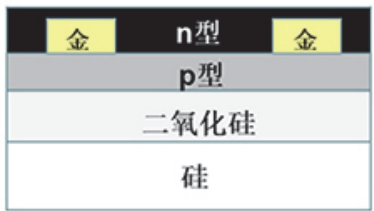

(d)

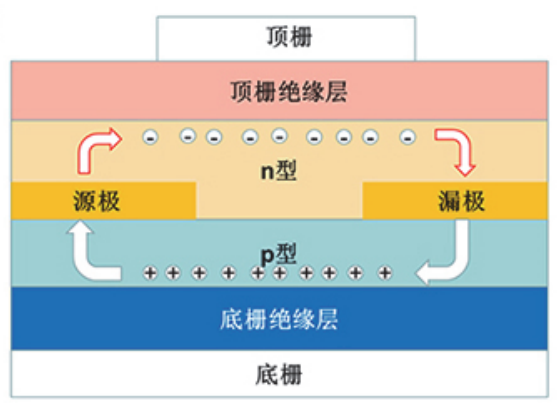

图 10 基于有机双层的双极性场效应晶体管的四种器件结构: (a)顶接触型, (b)底接触型, (c)中间接触型和(d)双栅极型 Figure 10 Four device structures of ambipolar OFETs based on organic bilayers: (a) top-contact, (b) bottom-contact, (c) middle-contact and (d) dual-gate type 
高迁移率且两种载流子传输性能相当的双极性有机场 效应晶体管的有效方法. 该器件结构中两种类型的半导 体分子需要具有一下特点: 首先, $\mathrm{p}$ 型有机半导体分子 的空穴迁移率和 $\mathrm{n}$ 型有机半导体分子的电子迁移率都要 高, 且两种载流子的迁移率要匹配; 其次, $p$ 型有机半导 体分子的 HOMO 能级与 $\mathrm{n}$ 型有机半导体分子的 LUMO 能级差越小越好, 这样使用一种金属电极可以同时匹配 两种载流子的功函, 有利于同时提高两种载流子的注入 效率，能极差低于 $2.0 \mathrm{eV}$ 以下时双极性晶体管一般会同 时展现出高的电子迁移率和高的空穴迁移率. 除此之 外, 电极材料、电极和有机半导体层之间的缓冲层、介 电层、有机半导体层的沉积顺序、有机半导体膜厚、测 试环境等因素都会影响基于有机双层的双极性有机场 效应晶体管的电学性能. 弄清楚这些影响因素与晶体管 的电学性能之间的关系, 能优化双极性晶体管的制备和 测试条件, 有利于获得高性能的双极性场效应晶体管. 希望这篇综述能对该领域的科学家提供帮助, 推动高性 能双极性有机场效应晶体管的发展及应用.

尽管双极性晶体管的器件制备和载流子迁移率已 被大量文献报道, 并取得了部分显著成果. 但是对于提 高双极性晶体管的开关比的研究还比较少. 目前已报道 的大多数双极性晶体管的开关比低于 $10^{2}$, 少数器件的 开关比达到 $10^{3}$, 远远低于单组份晶体管的器件开关比 $\left(10^{4} \sim 10^{7}\right)$. 双极性晶体管较低的开关比主要是由于在 栅压为零伏时, 带相反电荷的载流子会注入到导电沟道 中, 增大了开态电流, 降低了器件的电流开关比. 降低 双极性晶体管的电流开关比将是未来科学家们需要重 点关注的研究方向.

\section{References}

[1] (a) Sun, S.; Li, Y.; Zhang, S. Chin. Phys. B 2020, 29, 058503 (b) Jo, I. Y.; Park, J.-G.; Moon, J.-H.; Jung, J. Y.; Kim, D. E.; Baeg, K.-J. Org. Electron. 2019, 75, 105358.

(c) Kwon, H.; Yoo, H.; Nakano, M.; Takimiya, K.; Kim, J.-J.; Kim, J. K. RSC Adv. 2020, 10, 1910.

(d) Wang, L.; Wang, C.; Yu, X.; Zheng, L.; Zhang, X.; Hu, W. Sci. China Mater. 2020, 63, 122.

(e) Zhou, X.; Wang, Z.; Song, R. X.; Zhang, Y. D.; Zhu, L. A.; Xue, D.; Huang, L. Z.; Chi, L. F. J. Mater. Chem. C 2021, 9, 1584.

(f) Becharguia, H.; Mahdouani, M.; Bourguiga, R. Eur. Phys. J.-Appl. Phys. 2021, 93, 10.

(g) Wan, Y. J.; Deng, J.; Wu, W. L.; Zhou, J. D.; Niu, Q.; Li, H. Y.; Yu, H. K.; Gu, C.; Ma, Y. G. ACS Appl. Mater. Inter. 2020, 12, 43976.

[2] (a) Sonar, P.; Singh, S. P.; Li, Y.; Soh, M. S.; Dodabalapur, A. $A d v$. Mater. 2010, 22, 5409.

(b) Yuen, J. D.; Fan, J.; Seifter, J.; Lim, B.; Hufschmid, R.; Heeger, A. J.; Wudl, F. J. Am. Chem. Soc. 2011, 133, 20799.

(c) Chen, Z.; Lee, M. J.; Shahid Ashraf, R.; Gu, Y.; Albert-Seifried, S.; Meedom Nielsen, M.; Schroeder, B.; Anthopoulos, T. D.; Heeney, M.; McCulloch, I.; Sirringhaus, H. Adv. Mater. 2012, 24, 647.

(d) Lee, J.; Han, A. R.; Kim, J.; Kim, Y.; Oh, J. H.; Yang, C. J. Am.
Chem. Soc. 2012, 134, 20713.

(e) Fan, J.; Yuen, J. D.; Wang, M.; Seifter, J.; Seo, J.-H.; Mohebbi, A. R.; Zakhidov, D.; Heeger, A.; Wudl, F. Adv. Mater. 2012, 24, 2186.

[3] (a) Jiang, L.; Dong, H.; Hu, W. J. Mater. Chem. 2010, 20, 4994.

(b) Dong, H.; Fu, X.; Liu, J.; Wang, Z.; Hu, W. Adv. Mater. 2013, $25,6158$.

(c) Gao, X.; Zhao, Z. Sci. China Chem. 2015, 58, 947.

(d) Anthony, J. E.; Facchetti, A.; Heeney, M.; Marder, S. R.; Zhan, X. Adv. Mater. 2010, 22, 3876.

(e) Wang, C.; Dong, H.; Hu, W.; Liu, Y.; Zhu, D. Chem. Rev. 2012 112, 2208.

(f) Barłóg, M.; Zhang, X.; Kulai, I.; Yang, D. S.; Sredojevic, D. N.; Sil, A.; Ji, X.; Salih, K. S. M.; Bazzi, H. S.; Bronstein, H.; Fang, L.; Kim, J.; Marks, T. J.; Guo, X.; Al-Hashimi, M. Chem. Mater. 2019 31,9488 .

[4] Sun, B.; Hong, W.; Aziz, H.; Li, Y. Polym. Chem. 2015, 6, 938.

[5] (a) Lv, A.; Li, Y.; Yue, W.; Jiang, L.; Dong, H.; Zhao, G.; Meng, Q.; Jiang, W.; He, Y.; Li, Z.; Wang, Z.; Hu, W. Chem. Commun. 2012, 48,5154 .

(b) Lv, A.; Freitag, M.; Chepiga, K. M.; Schäfer, A. H.; Glorius, F.; Chi, L. Angew. Chem., Int. Ed. 2018, 57, 4792.

(c) Lv, A.; Stolte, M.; Würthner, F. Angew. Chem., Int. Ed. 2015, $127,10658$.

(d) Wen, J.; Xiao, C.; Lv, A.; Hayashi, H.; Zhang, L. Chem. Commun. 2018, 54, 5542.

(e) Luo, Y.; Yao, L.; Gu, W.; Xiao, C.; Liao, H.; Ravva, M. K.; Wang, Y.; Li, Z.; Zhang, L.; Lv, A.; Yue, W. Org. Electron. 2020, $85,105895$.

(f) Warren, P. R.; Hardigree, J. F. M.; Lauritzen, A. E.; Nelson, J.; Riede, M. AIP Adv. 2019, 9, 035202.

(g) Torres-Moya, I.; Carrillo, J. R.; Gomez, M. V.; Velders, A. H.; Donoso, B.; Rodriguez, A. M.; Diaz-Ortiz, A.; Navarrete, J. T. L.; Ortiz, R. P.; Prieto, P. Dyes Pigm. 2021, 191, 14.

(h) Nam, S.; Khim, D.; Martinez, G. T.; Varambhia, A.; Nellist, P. D.; Kim, Y.; Anthopoulos, T. D.; Bradley, D. D. C. Adv. Mater. 2021, 33, 2100421 .

[6] Dodabalapur, A.; Katz, H. E.; Torsi, L.; Haddon, R. C. Science 1995, 269, 1560

[7] (a) Shi, J. W.; Wang, H. B.; Song, D.; Tian, H. K.; Geng, Y. H.; Yan, D. H. Adv. Funct. Mater. 2007, 17, 397.

(b) An, M.-J.; Seo, H.-S.; Zhang, Y.; Oh, J.-D.; Choi, J.-H. Appl. Phys. Lett. 2010, 97, 023506.

[8] (a) Zaumseil, J.; Sirringhaus, H. Chem. Rev. 2007, 107, 1296.

(b) Chua, L.-L.; Zaumseil, J.; Chang, J.-F.; Ou, E. C. W.; Ho, P. K. H.; Sirringhaus, H.; Friend, R. H. Nature 2005, 434, 194.

(c) Cornil, J.; Brédas, J. L.; Zaumseil, J.; Sirringhaus, H. $A d v$. Mater. 2007, 19, 1791.

(d) Yang, C.-Y.; Dhananjay; Cheng, S.-S.; Ou, C.-W.; Chuang, Y.-C.; Wu, M.-C.; Chu, C.-W. Appl. Phys. Lett. 2008, 92, 253307.

(e) Yoon, M.-H.; Kim, C.; Facchetti, A.; Marks, T. J. J. Am. Chem. Soc. 2006, 128, 12851.

(f) Seo, J. H.; Chang, G. S.; Wilks, R. G.; Whang, C. N.; Chae, K. H.; Cho, S.; Yoo, K.-H.; Moewes, A. J. Phys. Chem. B 2008, 112, 16266.

[9] (a) Dodabalapur, A.; Katz, H. E.; Torsi, L.; Haddon, R. C. Appl. Phys. Lett. 1996, 68, 1108.

(b) Rost, C.; Gundlach, D. J.; Karg, S.; Rieß, W. J. Appl. Phys. 2004, 95, 5782 .

(c) Wang, J.; Wang, H.; Yan, X.; Huang, H.; Yan, D. Chem. Phys Lett. 2005, 407, 87.

[10] Obaidulla, S. M.; Giri, P. K. J. Mater. Chem. C 2015, 3, 7118.

[11] (a) Kim, D.-K.; Oh, J.-D.; Shin, E.-S.; Seo, H.-S.; Choi, J.-H. J. Appl. Phys. 2014, 115, 164503.

(b) Kang, S. J.; Yi, Y.; Kim, C. Y.; Cho, K.; Seo, J. H.; Noh, M.; Jeong, K.; Yoo, K.-H.; Whang, C. N. Appl. Phys. Lett. 2005, 87, 233502.

[12] (a) Ye, R.; Baba, M.; Oishi, Y.; Mori, K.; Suzuki, K. Appl. Phys. 
Lett. 2005, 86, 253505.

(b) Ye, R.; Baba, M.; Suzuki, K.; Mori, K. Appl. Surf. Sci. 2008, 254, 7885.

[13] Hu, Y.; Zhang, N.; Lin, J.; Qin, L.; Liu, X. Appl. Phys. Express 2012, 5, 095601.

[14] Zhu, X.; Zhang, Y.; Ren, X.; Yao, J.; Guo, S.; Zhang, L.; Wang, D.; Wang, G.; Zhang, X.; Li, R.; Hu, W. Small 2019, 15, 1902187.

[15] Yan, Y.; Sun, Q.-J.; Gao, X.; Deng, P.; Zhang, Q.; Wang, S.-D. Appl. Phys. Lett. 2013, 103, 073303.

[16] Imai, S.; Yanagi, H.; Hotta, S. Org. Electron. 2013, 14, 80.

[17] Janasz, L.; Marszalek, T.; Zajaczkowski, W.; Borkowski, M.; Goldeman, W.; Kiersnowski, A.; Chlebosz, D.; Rogowski, J.; Blom, P.; Ulanski, J.; Pisula, W. J. Mater. Chem. C 2018, 6, 7830.

[18] Yan, H.; Kagata, T.; Okuzaki, H. Appl. Phys. Lett. 2009, 94, 023305 .

[19] (a) Huang, S.; Peng, B.; Chan, P. K. L. Adv. Electron. Mater. 2017, 3, 1700268 .

(b) Wei, Z.; Xu, W.; Hu, W.; Zhu, D. Langmuir 2009, 25, 3349.

(c) Kuwahara, E.; Kubozono, Y.; Hosokawa, T.; Nagano, T.; Masunari, K.; Fujiwara, A. Appl. Phys. Lett. 2004, 85, 4765.

[20] Di Girolamo, F. V.; Barra, M.; Chiarella, F.; Lettieri, S.; Salluzzo, M.; Cassinese, A. Phys. Rev. B 2012, 85, 125310.

[21] Loi, M. A.; Rost-Bietsch, C.; Murgia, M.; Karg, S.; Riess, W.; Muccini, M. Adv. Funct. Mater. 2006, 16, 41.

[22] (a) Lee, J.; Han, A. R.; Yu, H.; Shin, T. J.; Yang, C.; Oh, J. H. J. Am. Chem. Soc. 2013, 135, 9540 .

(b) Sun, B.; Hong, W.; Yan, Z.; Aziz, H.; Li, Y. Adv. Mater. 2014, 26, 2636.

(c) Xiao, C.; Zhao, G.; Zhang, A.; Jiang, W.; Janssen, R. A. J.; Li, W.; Hu, W.; Wang, Z. Adv. Mater. 2015, 27, 4963.

(d) Gao, Y.; Zhang, X.; Tian, H.; Zhang, J.; Yan, D.; Geng, Y.; Wang, F. Adv. Mater. 2015, 27, 6753.

(e) Liu, J.; Liu, J.; Zhang, J.; Li, C.; Cui, Q.; Teng, F.; Li, H.; Jiang, L. J. Mater. Chem. C 2020, $8,4303$.

[23] Xiong, N.; Xiao, P.; Li, M.; Xu, H.; Yao, R.; Wen, S.; Peng, J. Appl. Phys. Lett. 2013, 102, 242102.

[24] (a) Dimitrakopoulos, C. D.; Malenfant, P. R. L. Adv. Mater. 2002, 14, 99.

(b) Katz, H. E. Chem. Mater. 2004, 16, 4748.

[25] Pal, B. N.; Trottman, P.; Sun, J.; Katz, H. E. Adv. Funct. Mater. 2008, 18, 1832 .

[26] Yang, C.; Kwack, Y.; Kim, S. H.; An, T. K.; Hong, K.; Nam, S.; Park, M.; Choi, W.-S.; Park, C. E. Org. Electron. 2011, 12, 411.

[27] (a) Koo, J. B.; Ku, C. H.; Lim, S. C.; Kim, S. H.; Lee, J. H. Appl. Phys. Lett. 2007, 90, 133503.

(b) Kim, S. H.; Jang, J.; Jeon, H.; Yun, W. M.; Nam, S.; Park, C. E. Appl. Phys. Lett. 2008, 92, 183306.

[28] Nakanotani, H.; Yahiro, M.; Adachi, C.; Yano, K. Appl. Phys. Lett. 2007, 90, 262104.

[29] Sun, S.; Lan, L.; Li, Y.; Ning, H.; Yao, R.; Wang, L.; Peng, J. RSC Adv. 2017, 7, 5966.

[30] (a) Singh, T. B.; Senkarabacak, P.; Sariciftci, N. S.; Tanda, A.; Lackner, C.; Hagelauer, R.; Horowitz, G. Appl. Phys. Lett. 2006, 89, 033512 .

(b) An, M.-J.; Seo, H.-S.; Zhang, Y.; Oh, J.-D.; Choi, J.-H. Appl. Phys. Lett. 2010, 97, 023506.

(c) Oh, J.-D.; Kim, J.-W.; Kim, D.-K.; Choi, J.-H. Org. Electron. 2016, 30, 131 .

[31] He, X. X.; Chow, W.; Liu, F. C.; Tay, B.; Liu, Z. Small 2017, 13, 1602558 .

[32] Smith, J.; Bashir, A.; Adamopoulos, G.; Anthony, J. E.; Bradley, D. D. C.; Heeney, M.; McCulloch, I.; Anthopoulos, T. D. Adv. Mater. 2010, 22, 3598

[33] Li, M.; Wang, J.; Cai, X.; Liu, F.; Li, X.; Wang, L.; Liao, L.; Jiang, C. Adv. Electron. Mater. 2018, 4, 1800211.

[34] Zhou, Y.; Han, S.-T.; Zhou, L.; Yan, Y.; Huang, L.-B.; Huang, J.; Roy, V. A. L. J. Mater. Chem. C 2013, 1, 7073.

[35] Sun, S.; Li, Y.; Lan, L.; Xiao, P.; Chen, Z.; Lin, Z.; Chen, J.; Peng,
J.; Cao, Y. Org. Electron. 2017, 43, 162.

[36] Wei, Z.; Xu, W.; Hu, W.; Zhu, D. J. Mater. Chem. 2008, 18, 2420.

[37] Tang, Q.; Tong, Y.; Li, H.; Ji, Z.; Li, L.; Hu, W.; Liu, Y.; Zhu, D. Adv. Mater. 2008, 20, 1511.

[38] Lu, C.; Wang, J.; Chang, H.-C.; Chiu, Y.-C.; Chen, H.-Y.; Wu, H.-C.; Higashihara, T.; Chen, W.-C. J. Mater. Chem. C 2014, 2, 7489.

[39] Zhang, X.; Mao, J.; Deng, W.; Xu, X.; Huang, L.; Zhang, X.; Lee, S.-T.; Jie, J. Adv. Mater. 2018, 30, 1800187.

[40] Zhang, Y.; Liu, J.; Nguyen, T.-Q. ACS Appl. Mater. Inter. 2013, 5, 2347.

[41] Szendrei, K.; Jarzab, D.; Chen, Z.; Facchetti, A.; Loi, M. A. J. Mater. Chem. 2010, 20, 1317.

[42] Meijer, E. J.; de Leeuw, D. M.; Setayesh, S.; van Veenendaal, E.; Huisman, B. H.; Blom, P. W. M.; Hummelen, J. C.; Scherf, U.; Klapwijk, T. M. Nat. Mater. 2003, 2, 678.

[43] Rost, C.; Karg, S.; Riess, W.; Loi, M. A.; Murgia, M.; Muccini, M. Appl. Phys. Lett. 2004, 85, 1613.

[44] (a) Tandy, K.; Dutta, G. K.; Zhang, Y.; Venkatramaiah, N.; Aljada, M.; Burn, P. L.; Meredith, P.; Namdas, E. B.; Patil, S. Org. Electron. 2012, 13, 1981 .

(b) Lee, H.-S.; Lee, J. S.; Cho, S.; Kim, H.; Kwak, K.-W.; Yoon, Y.; Son, S. K.; Kim, H.; Ko, M. J.; Lee, D.-K.; Kim, J. Y.; Park, S.; Choi, D. H.; Oh, S. Y.; Cho, J. H.; Kim, B. J. Phys. Chem. C 2012, 116, 26204.

(c) Ribierre, J. C.; Ghosh, S.; Takaishi, K.; Muto, T.; Aoyama, T. J. Phys. D: Appl. Phys. 2011, 44, 205102.

[45] Horlet, M.; Kraus, M.; Brütting, W.; Opitz, A. Appl. Phys. Lett. 2011, 98, 233304.

[46] (a) Schidleja, M.; Melzer, C.; Seggern, H. V. Appl. Phys. Lett. 2009, 94, 123307.

(b) Wang, Y.; Liu, D.; Ikeda, S.; Kumashiro, R.; Nouch, R.; Xu, Y.; Shang, H.; Ma, Y.; Tanigaki, K. Appl. Phys. Lett. 2010, 97, 033305. (c) Schmechel, R.; Ahles, M.; Von Seggern, H. J. Appl. Phys. 2005, 98, 084511 .

(d) Opitz, A.; Horlet, M.; Kiwull, M.; Wagner, J.; Kraus, M.; Brütting, W. Org. Electron. 2012, 13, 1614

[47] (a) Yoo, D.; Hasegawa, T.; Kohara, A.; Sugiyama, H.; Ashizawa, M.; Kawamoto, T.; Masunaga, H.; Hikima, T.; Ohta, N.; Uekusa, H.; Matsumoto, H.; Mori, T. Dyes Pigments 2020, 180, 108418. (b) Opoku, H.; Bathula, C.; Mamo, M. D.; Shrestha, N. K.; Lee, T.; Noh, Y.-Y. Macromol. Res. 2019, 27, 90.

(c) Parka, S.; Leeb, B.; Baeb, B.; Chaib, J.; Leeb, S.; Kima, C. Synth. Met. 2019, 253, 40.

[48] Long, D. X.; Noh, Y.-Y. J. Nanosci. Nanotechnol. 2017, 17, 5709.

[49] Park, J.; Kim, M.; Yeom, S. W.; Ha, H. J.; Song, H.; Min Jhon, Y.; Kim, Y. H.; Ju, B. K. Nanotechnology 2016, 27, 225302.

[50] (a) Khim, D.; Baeg, K.-J.; Kim, J.; Yeo, J.-S.; Kang, M.; Amegadzea, P. S. K.; Kim, M.-G.; Cho, J.; Lee, J. H.; Kim, D.-Y.; Noh, Y.-Y. J. Mater. Chem. 2012, 22, 16979.

(b) Baeg, K. J.; Kim, J.; Khim, D.; Caironi, M.; Kim, D. Y.; You, I. K.; Quinn, J. R.; Facchetti, A.; Noh, Y. Y. ACS Appl. Mater. Interfaces 2011, 3, 3205.

[51] (a) Xu, Y.; Baeg, K. J.; Park, W. T.; Cho, A.; Choi, E. Y.; Noh, Y. Y. ACS Appl. Mater. Inter. 2014, 6, 14493.

(b) Chen, H.; Guo, Y.; Mao, Z.; Yu, G.; Huang, J.; Zhao, Y.; Liu, Y. Chem. Mater. 2013, 25, 3589.

[52] Ito, Y.; Virkar, A. A.; Mannsfeld, S.; Oh, J. H.; Toney, M.; Locklin, J.; Bao, Z. J. Am. Chem. Soc. 2009, 131, 9396.

[53] Baeg, K. J.; Khim, D.; Jung, S. W.; Kang, M.; You, I. K.; Kim, D. Y.; Facchetti, A.; Noh, Y. Y. Adv. Mater. 2012, 24, 5433.

[54] Kobayashi, S.; Nishikawa, T.; Takenobu, T.; Mori, S.; Shimoda, T.; Mitani, T.; Shimotani, H.; Yoshimoto, N.; Ogawa, S.; Iwasa, Y. Nat. Mater. 2004, 3, 317.

[55] Ji, Y.; Xiao, C.; Heintges, G. H. L.; Wu, Y.; Janssen, R. A. J.; Zhang, D.; Hu, W.; Wang, Z.; Li, W. J. Polym. Sci. Polym. Chem. 2016, 54, 34.

[56] Yang, J.; Zhao, Z.; Geng, H.; Cheng, C.; Chen, J.; Sun, Y.; Shi, L.; 
Yi, Y.; Shuai, Z.; Guo, Y.; Wang, S.; Liu, Y. Adv. Mater. 2017, 29, 1702115 .

[57] Lei, T.; Dou, J. H.; Cao, X. Y.; Wang, J. Y.; Pei, J. Adv. Mater. 2013, 25, 6589.

[58] Kim, M.; Park, W.-T.; Park, S. A.; Park, C. W.; Ryu, S. U.; Lee, D. H.; Noh, Y.-Y.; Park, T. Adv. Funct. Mater. 2019, 29, 1805994.

[59] Di, C.; Liu, Y.; Yu, G.; Zhu, D. Acc. Chem. Res. 2009, 42, 1573.

[60] (a) Nénon, S.; Kanehira, D.; Yoshimoto, N.; Fages, F.; Videlot-Ackermann, C. Synth. Met. 2011, 161, 1915.

(b) Wang, S. D.; Kanai, K.; Ouchi, Y.; Seki, K. Org. Electron. 2006, $7,457$.

[61] (a) Zhao, G.; Cheng, X.-M.; Tian, H.-J.; Du, B.-Q.; Liang, X.-Y.;
Wu, F. Acta Phys. Sin. 2012, 61, 218502.

(b) Morita, T.; Singh, V.; Oku, S.; Nagamatsu, S.; Takashima, W.; Hayase, S.; Kaneto, K. Jpn. J. Appl. Phys. 2010, 49, 041601.

(c) Sarkar, T.; Vinokur, J.; Shamieh, B.; Savikhin, V.; Toney, M. F.; Frey, G. L. Chem. Mater. 2019, 31, 7046.

[62] Lee, G.; Kim, M.-H.; Noh, S.-P.; Keum, C.-M.; Lee, S.-D. Mol. Cryst. Liq. Cryst. 2014, 597, 8.

[63] Lin, H.-W.; Lee, W.-Y.; Chen, W.-C. J. Mater. Chem. 2012, 22, 2120.

[64] Lee, D. Y.; Tamilavan, V.; Shin, I.; Lee, J.; Jung, Y. K.; Jeong, J. H.; Cho, S.; Lee, B. R.; Park, S. H. Polym. J. 2020, 52, 581. 